\title{
VERTICAL DYNAMIC LOADS \\ ON FOOTBRIDGES GENERATED \\ BY PEOPLE RUNNING
}

2020/15(1)

MAREK PAŃTAK ${ }^{*}$

Division of Bridge, Metal and Timber Structures, Faculty of Civil Engineering, Cracow University of Technology, Cracow, Poland

Received 5 November 2018; accepted 11 September 2019

\begin{abstract}
This paper concerns the issue of the dynamic impact of people running on footbridges with particular with attention to various footstrike patterns occurring during the running (i.e. heel strike pattern and forefoot strike pattern). The results of a series of laboratory tests of vertical ground reaction force $(V G R F)$ measurements generated by running people are presented along with the characteristics of the VGRF curves. Based on the results of the tests, a new proposal for a dynamic load model generated by people classified as heel strike runners has been developed, and corrections of the input parameters of two load models proposed by other authors have been performed. Moreover, the VGRF modelling technique using the Gaussian functions is presented along with a set of equations describing the variability of the Gaussian function parameters as a function of the frequency of running. The presented methods of the VGRF modelling allow increasing the accuracy of determining the VGRF values and, consequently, increasing the accuracy of dynamic analyses of footbridges subjected to dynamic loads generated by people running.
\end{abstract}

Keywords: dynamic load, footbridge vibration, ground reaction forces, jogging, running, structural vibration assessment, vibration serviceability limit state.

\footnotetext{
* Corresponding author. E-mail: mpantak@pk.edu.pl

Marek PAŃTAK (ORCID ID 0000-0003-4807-4212) 


\section{Introduction}

The fundamental vibration frequency of medium span footbridges often appears in the frequency range of $2.20-3.40 \mathrm{~Hz}$. This frequency range is also the range of the frequency of steps during running. The synchronisation of step frequency with the natural vibration frequency of the footbridge may cause excessive vibration of the structure. This synchronisation can often be observed in situations of the activity of a person running through the footbridge or during occasional sports events, e.g. city marathons or half marathons. The results of dynamic field tests performed by the author on thirty-one footbridges show that $55 \%$ of the structures are subject to a natural vibration frequency in the range of the frequency of steps during running (Figure 1a).

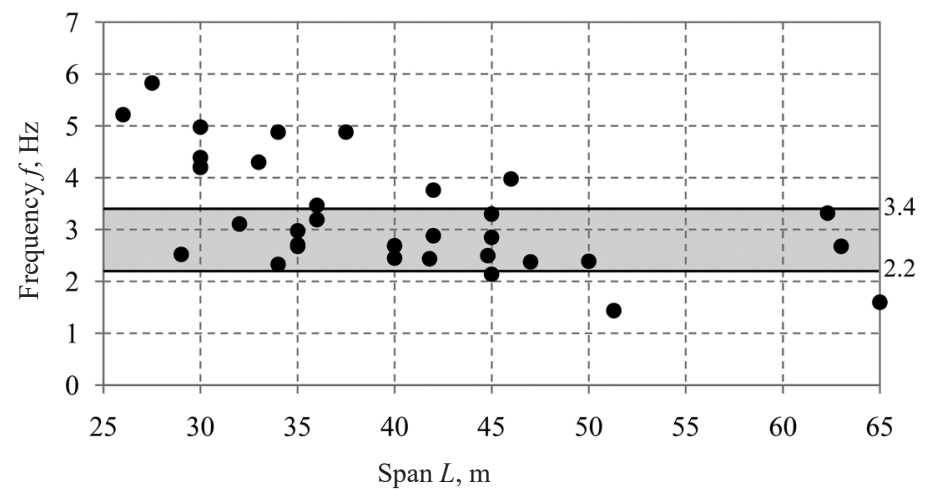

a)

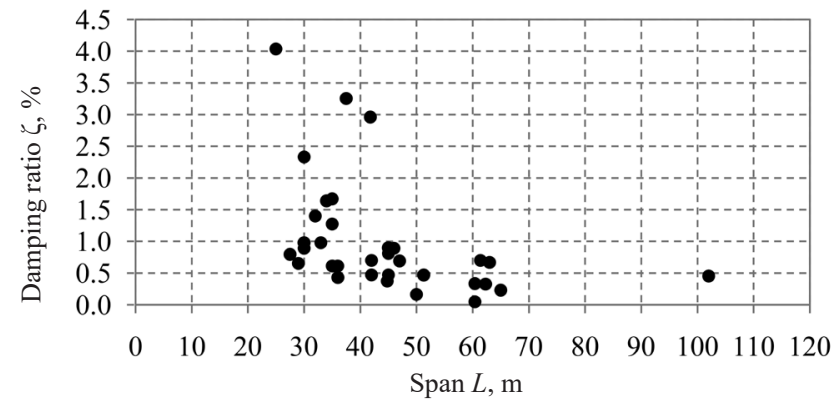

b)

Figure 1. Selected dynamic parameters of medium span footbridges a) fundamental natural vertical vibration frequencies (shaded area - the range of frequency of steps during running), b) damping ratio in steel footbridges 
The amplitude of vibration (vibration acceleration) induced in the case of the synchronisation of the step frequency of a running person with the natural vibration frequency of the structure can reach a high value of up to around $a=4.0 \mathrm{~m} / \mathrm{s}^{2}$ or more, especially in the case of steel footbridges characterised by low damping $\zeta \approx 0.005$ (Figure $1 \mathrm{~b}$ ). These high amplitude vibrations, which can be caused only by one running person, can strongly disturb the walking of other users passing over the footbridge. During these vibrations, pedestrians' feet are bounced by the vibrating footbridge deck, and walking is difficult, vibrations are unpleasant and often force walking users to stop and wait for the reduction or expiration of the vibrations.

A vertical ground reaction force $(V G R F)$ is the main force component arising during running. The amplitude of the normalised (dimensionless) $V G R F$ defined as a $V G R F / G$ (where $G=m g, m$ - bodyweight of running person, $\mathrm{kg}, g$ - acceleration of gravity, $\mathrm{m} / \mathrm{s}^{2}$ ) induced during recreational running typically reaches the value of $2.0-2.5(V G R F / G \geq 3.0$ can be reached during sprint) (Clark \& Weyand, 2014; Nilsson \& Thorstensson, 1989).

The time course of the $V G R F$ depends on the running technique, which manifests itself in different foot strike patterns (foot-ground contact patterns). The three general foot strike patterns that can be observed during running are: heel strike pattern (also known as a rearfoot strike pattern), in which the heel makes contact with the ground first; midfoot strike in which the outside edge of the whole foot contacts the ground first; forefoot strike, in which the outside edge of the forefoot contacts the ground first (Figure 2). Most recreational runners, about 90-95\%, are the heel strike runners (Almeida, Saragiotto, Yamato, \& Lopes, 2015; Bakkie et al., 2013; Larson et al., 2011). As reported in (Hasegawa, Yamauchi, \& Kraemer, 2007), in a group of professional marathon runners, nearly $75 \%$ of runners were also heel strike runners. The heel strike style can be considered to be the most common running style, accounting for $75-95 \%$ of runners. Despite this, the existing models of the VGRF generated by running people are mainly related to forces generated by forefoot strike runners (e.g. models proposed by (Bachmann et al., 1995; Occhiuzzi, Spizzuoco, \& Ricciardelli, 2008)) without taking into account the occurrence of the impact peak in the $V G R F$ generated by heel strike runners (Figure 2).

It is worth noting that the VGRF generated by the forefoot strike runners are characterised by lower contact time and higher amplitude than forces induced by heel strike runners (compare Table 1 and Table 2). For this reason, the dynamic response of the structure determined using the $V G R F$ generated by the forefoot strike runners 
has the most significant value (conservative approach). However, this can result in an inaccurate picture of how the structure behaves in the situation when the knowledge of the real dynamic response of the structure is desired (when the serviceability limit state requirements are verified). The use of only the VGRF models defined for forefoot strike runners, accounting for $5-25 \%$ of runners, in the dynamic analyses can lead to overestimation of the vibration of the structure, e.g. in the analysis of the dynamic impact of a group of runners. A new model of the $V G R F$ generated by the heel strike runners will increase the accuracy of estimation of the dynamic response of the structure. In addition, more accurate load models can also be used in the design phase of vibration dampers to check and, if necessary, increase the accuracy of tuning of vibration damper parameters.

The VGRF generated by a heel strike runner is characterised by two peaks: an impact peak (the first small peak) and a propulsive peak (the second big peak, also known as the active peak) (Figure 2). During the forefoot strike running only one active peak occurs. The midfoot strike running is an intermediate case, in which the VGRF curve is similar to forefoot strike running without an apparent impact peak, only a slight disturbance in the course of the VGRF may occur. In all cases, the active peak occurs when the body centre of mass moves over the foot.

In order to perform a detailed analysis of the $V G R F$ generated during different foot ground contact patterns and to develop a more accurate

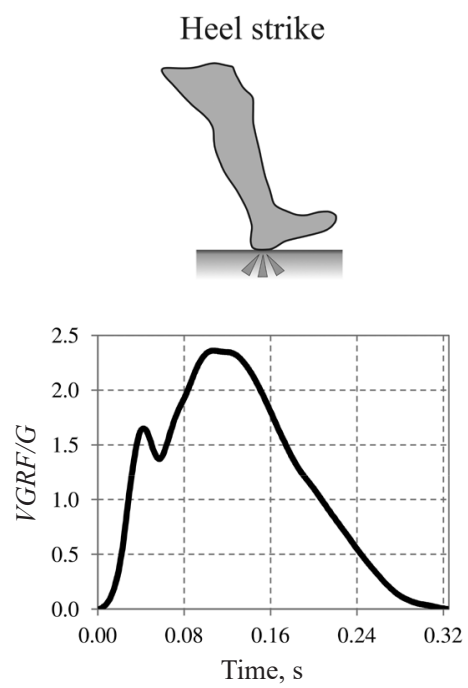

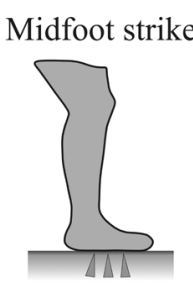

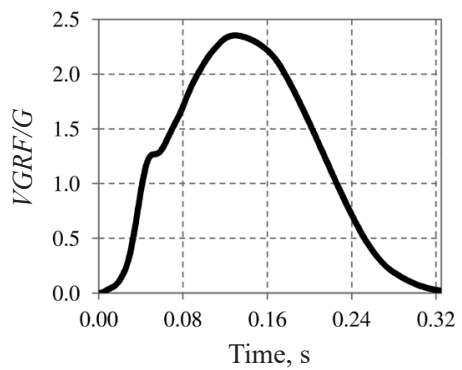

Forefoot strike
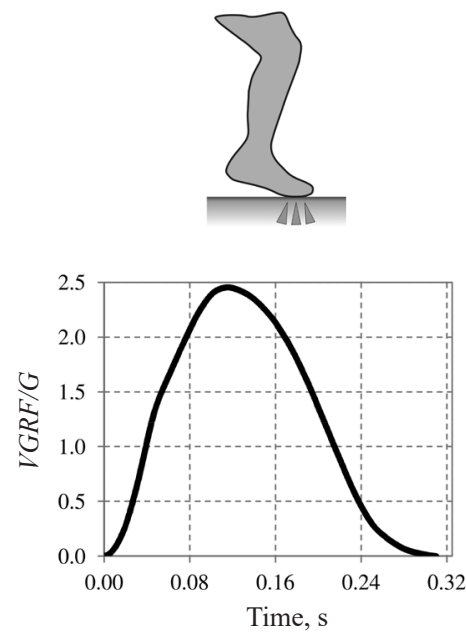

Figure 2. Foot strike patterns during running and corresponding normalised vertical ground reaction forces (VGRF/G) 
mathematical model of the $V G R F$ generated during the most common Running running technique, i.e. heel-strike running, a series of laboratory tests of the $V G R F$ measurements were performed.

\section{Experiment}

\subsection{Force measurement}

The force measurements were performed in laboratory conditions using two AMTI 6-degree of freedom force plates $(40 \mathrm{~cm} \times 60 \mathrm{~cm})$. During laboratory tests, the AMTI force plates were mounted one by one (in-line) centrally within the rigid ten-metre-long walkway (Figure 3).
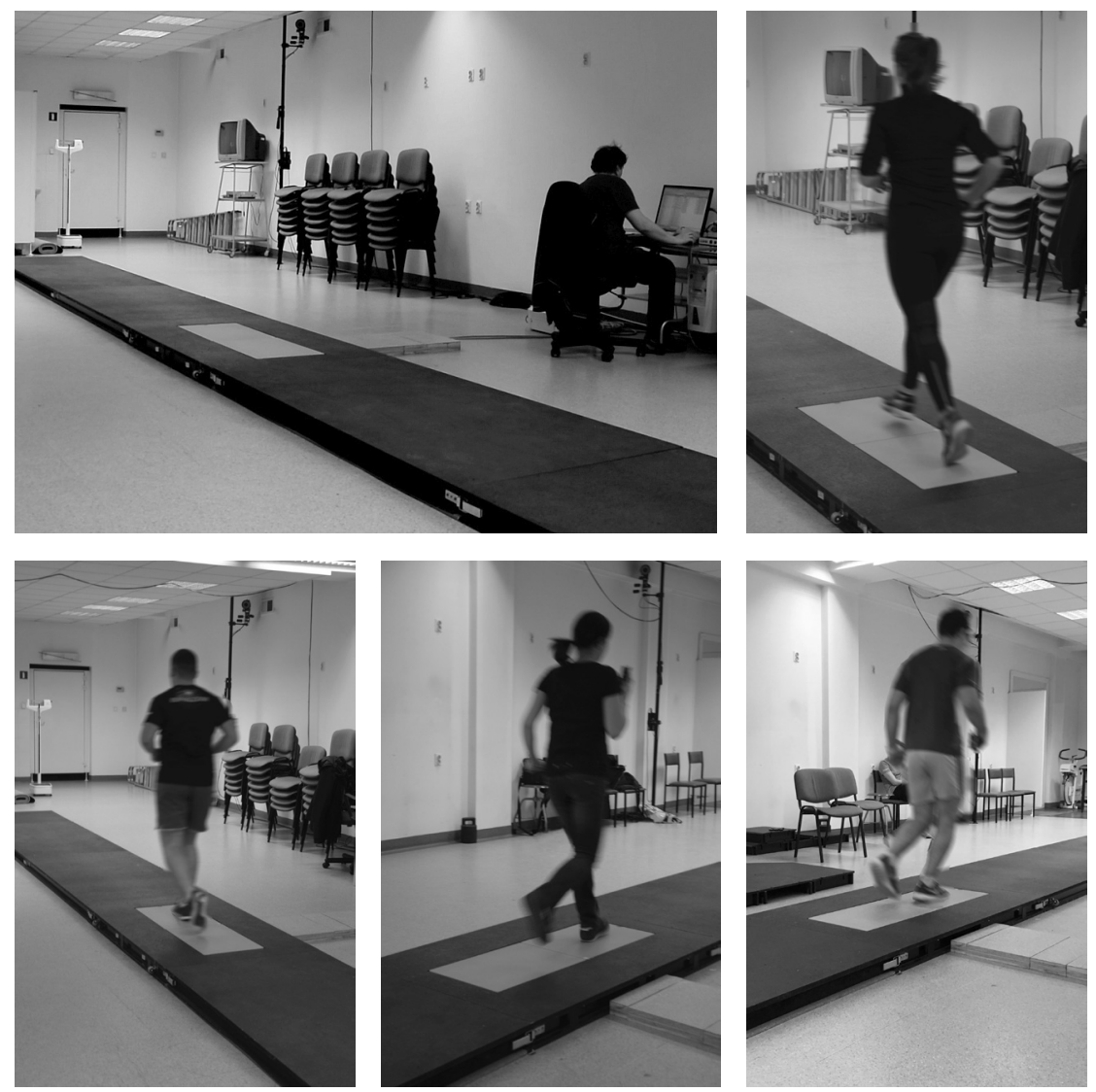

Figure 3. Laboratory tests: general view of the walkway with centrally placed force plates, examples of running volunteers 
The data were collected with a sample rate of $1000 \mathrm{~Hz}$ (a high sampling rate was necessary to accurately measure the impact peak of the VGRF generated by heel strike runners).

During the tests, a group of 13 healthy volunteers (with full mobility enabling the realisation of the assumed research methodology, without injury and disabilities) was examined ( 5 males and 8 females, aged 22-45 years (mean \pm SD: $25 \pm 6.8$ years), weight $51.6-108.4 \mathrm{~kg}$ (mean \pm SD: $70.2 \mathrm{~kg} \pm 16.2 \mathrm{~kg}$ ), height 158-187 cm (mean \pm SD: $172.3 \mathrm{~m}$ $\pm 9.8 \mathrm{~m}$ )). Volunteers were chosen from the 18-45 age group (a social group characterised by a high percentage of runners (Scheerder, Breedveld, \& Borgers, 2015; Sołtys et al., 2014) by convenience sampling method (non-probability sampling method): among students and employees of the University of Physical Education in Cracow and Cracow University of Technology.

The laboratory tests were performed in the frequency range $2.40-3.40 \mathrm{~Hz}$ to simulate slow, moderate and fast running pace. The assumed frequency increment was $0.2 \mathrm{~Hz}$. It was assumed that the examined range of the frequency of steps during running occurs on footbridges during recreational running and marathon events. Cases of very fast running or sprinting were not studied.

During the tests, the test participants ran in sport's shoes. All participants were asked to run at the pace indicated by the electronic metronome (the running pace was recalculated from $\mathrm{Hz}$ to BPM (beats per minute)) and to try to hit the region of the force plates with their feet. Before force measurement, each person practised running with the indicated frequency to better match the running pace during the final measurement. All test participants performed two series of running for each frequency. The duration of each series was $60 \mathrm{~s}$. After two series, the examined person rested during the series performed by other participants of the studies. Next, the frequency was increased by a step of $0.20 \mathrm{~Hz}$, and the measurement was repeated for the new frequency.

The results obtained during the study coincided with the results obtained in independent studies by other researchers (e.g. Racic \& Morin, 2014; Nilsson \& Thorstensson, 1989; Clark \& Weyand, 2014). Considering compatibility, the size of the group of volunteers was acknowledged as sufficient and suitable to examine the time course of the VGRFs. The examined group of volunteers allowed generating a large number of samples of the VGRFs for each tested frequency of running (see Table 1 and 2). On this basis, the statistical relevance of the employed data was acknowledged as appropriate to investigate the variability of the VGRFs parameters. The new data did not bring significant changes to the theoretical reconstruction of the tested physical quantity. 


\subsection{Data processing}

In Figure 4, example charts of the normalised $V G R F / G$ forces generated by heel-strike and forefoot runners are presented. The charts correspond to two different runners with comparable body weight $51.6 \mathrm{~kg}$ and $54.3 \mathrm{~kg}$. Visible differences in the amplitude of the presented forces (dimensionless amplitude $V G R F / G$ ) result from the running technique. Forefoot running is characterised by a higher amplitude of force and shorter contact time (Table 2).

The normalised $V G R F / G$ forces were analysed to parameterise the curve by the following indicators: the normalised (dimensionless) amplitude of the impact peak $\left(A_{i p}\right)$; the normalised (dimensionless) amplitude of the propulsive peak ( $A_{p p, h}$ or $\left.A_{p p, f}\right)$; the approximated contact time of the foot with the ground $\left(t_{c h, a}\right.$ or $\left.t_{c f, a}\right)$; the time of occurrence of $A_{i p}\left(t_{i p}\right)$ and time of occurrence of $A_{p p, h}$ or $A_{p p, f}\left(t_{p p, h}\right.$ or $\left.t_{p p, f}\right)$ (Figure 5). Approximated contact time of the foot with the ground (indicated by a dashed line in Figure 5) was assumed as a curve indicator due to the method of force modelling (see Section 2.1), which precluded reconstruction of the actual curvature of the graph in its final part (omitted low values of the VGRF/G were assumed negligible for dynamic analyses of footbridges).

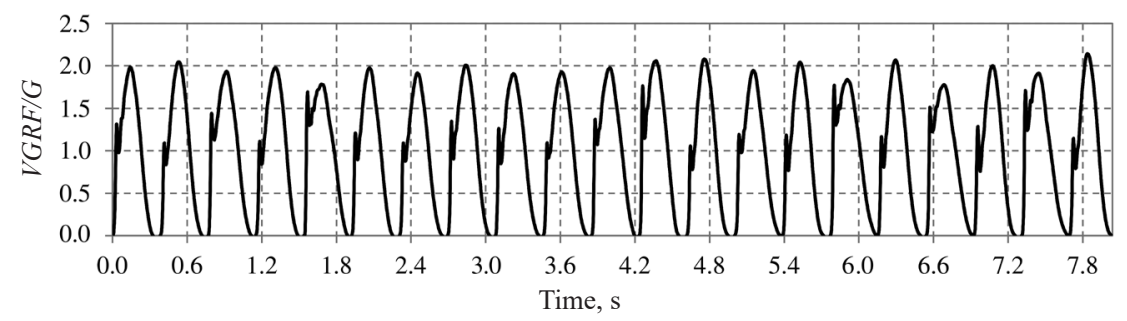

a)

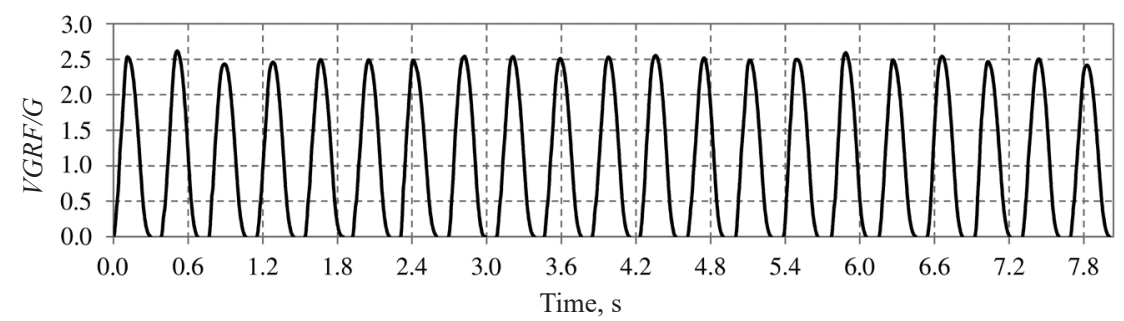

b)

Figure 4. Example charts of the normalised VGRF/G forces generated by a) a heel strike runner $\left(f_{r}=2.60 \mathrm{~Hz}, G=51.6 \mathrm{~kg}\right)$, b) a forefoot strike runner $\left(f_{r}=2.60 \mathrm{~Hz}, \mathrm{G}=54.3 \mathrm{~kg}\right)$ 
In Figures $5 \mathrm{c}$ and $5 \mathrm{~d}$, the frequency content of the $V G R F / G$ for heelstrike and forefoot strike running technique are also presented. In case of heel-strike running technique, a large number of harmonics (about 15-20 harmonics in frequency range up to $40.0 \mathrm{~Hz}(\max 60.0 \mathrm{~Hz}$ )

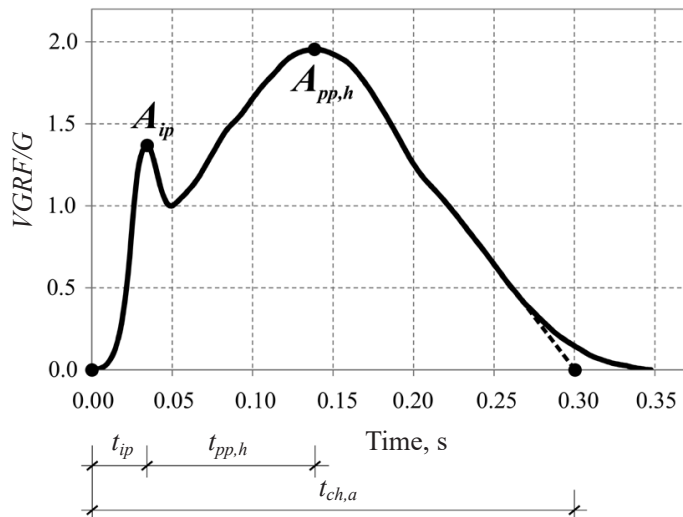

a)

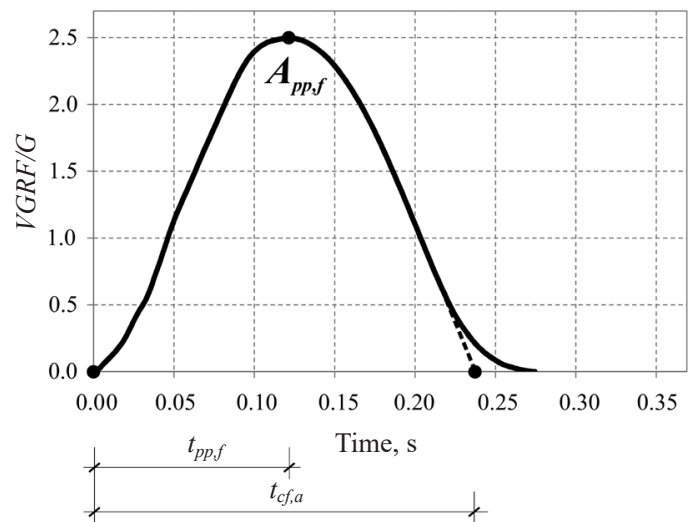

b)

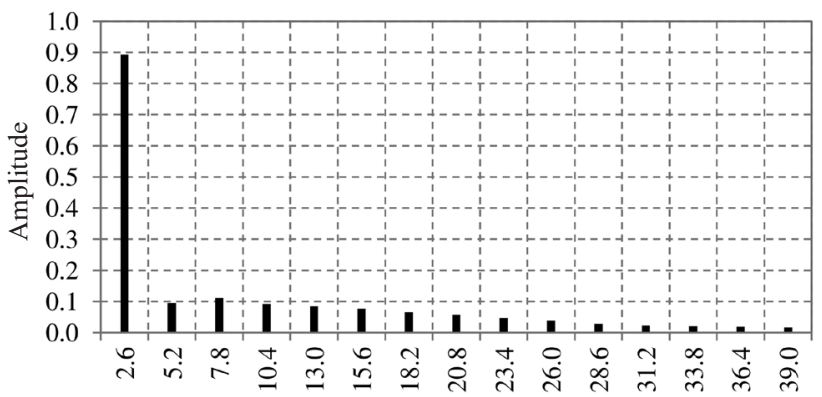

c) Frequency, Hz

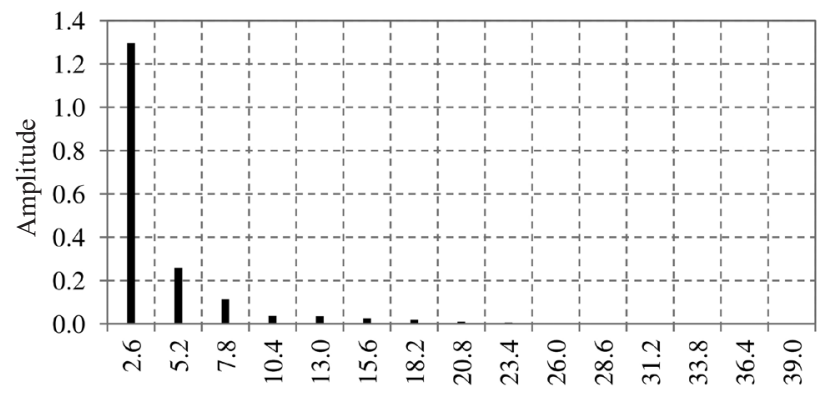

d)

Figure 5. The VGRF/G curve indicators and example of the frequency content of the VGRF/G for $a$ ), c) heel-strike running technique, b), d) forefoot strike running technique 
Table 1. The VGRF/G curve indicators - heel-strike running

(mean value \pm standard deviation (SD))

\begin{tabular}{|c|c|c|c|c|c|c|}
\hline \multirow{2}{*}{ 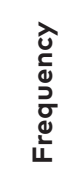 } & \multirow{2}{*}{$\begin{array}{l}\text { The number } \\
\text { of } V G R F \\
\text { samples } \\
\text { (heel strike } \\
\text { pattern) }\end{array}$} & \multicolumn{5}{|c|}{$\begin{array}{c}\text { Indicator } \\
(\text { mean } \pm \text { standard deviation })\end{array}$} \\
\hline & & $A_{i p} \pm S D$ & $A_{p p, h} \pm S D$ & $t_{i p} \pm S D, s$ & $t_{p p, h} \pm S D, s$ & $t_{c h, a} \pm \mathrm{SD}, \mathrm{s}$ \\
\hline 2.40 & 111 & $1.245 \pm 0.130$ & $2.047 \pm 0.178$ & $0.048 \pm 0.008$ & $0.153 \pm 0.012$ & $0.320 \pm 0.019$ \\
\hline 2.60 & 114 & $1.311 \pm 0.225$ & $2.155 \pm 0.171$ & $0.044 \pm 0.008$ & $0.134 \pm 0.011$ & $0.281 \pm 0.015$ \\
\hline 2.80 & 139 & $1.280 \pm 0.215$ & $2.203 \pm 0.192$ & $0.041 \pm 0.006$ & $0.121 \pm 0.014$ & $0.254 \pm 0.017$ \\
\hline 3.00 & 132 & $1.255 \pm 0.212$ & $2.180 \pm 0.176$ & $0.041 \pm 0.008$ & $0.114 \pm 0.013$ & $0.240 \pm 0.017$ \\
\hline 3.20 & 76 & $1.345 \pm 0.210$ & $2.098 \pm 0.181$ & $0.038 \pm 0.007$ & $0.107 \pm 0.009$ & $0.235 \pm 0.008$ \\
\hline 3.40 & 80 & $1.346 \pm 0.147$ & $2.085 \pm 0.191$ & $0.038 \pm 0.005$ & $0.103 \pm 0.012$ & $0.222 \pm 0.015$ \\
\hline
\end{tabular}

have a significant relevance for the accuracy of the VGRF curve fitting (harmonics with a frequency greater than $10 \mathrm{~Hz}$ are important for the correct reconstruction of the impact peak occurring on the VGRF curve). In case of forefoot running technique, the use of about 5-7 harmonics (min 3 harmonics) in the frequency range up to $18.0 \mathrm{~Hz}$ (max $20.0 \mathrm{~Hz}$ ) reconstructing the VGRF curve accurately. Similar results were also presented by other authors (Gruber, Davis, \& Hamill, 2011; Hamill \& Gruber, 2017; Gruber, Edwards, Hamill, Derrick, \& Boyer, 2017).

In Tables 1 and 2, the VGRF/G curve indicators identified for heelstrike running and forefoot strike running, respectively, are presented along with the number of the analysed $V G R F / G$ samples characterised by the corresponding foot strike pattern.

Table 2. The VGRF/G curve indicators - forefoot strike running (mean value \pm standard deviation (SD))

\begin{tabular}{ccccc}
\hline \multirow{2}{*}{ Frequency } & \multirow{2}{*}{$\begin{array}{c}\text { The number } \\
\text { of VGRF samples } \\
\text { (forefoot strike pattern) }\end{array}$} & \multicolumn{3}{c}{$\begin{array}{c}\text { Indicator } \\
\text { (mean } \pm \text { standard deviation) }\end{array}$} \\
\cline { 3 - 5 } & 96 & $\boldsymbol{A}_{p p, f} \pm$ SD & $\boldsymbol{t}_{p p, f} \pm$ SD, s & $\boldsymbol{t}_{c f, a} \pm$ SD, s \\
\hline 2.40 & 124 & $2.300 \pm 0.127$ & $0.125 \pm 0.013$ & $0.283 \pm 0.009$ \\
2.60 & 106 & $2.385 \pm 0.124$ & $0.114 \pm 0.011$ & $0.253 \pm 0.006$ \\
2.80 & 127 & $2.453 \pm 0.155$ & $0.104 \pm 0.012$ & $0.226 \pm 0.005$ \\
3.00 & 189 & $2.450 \pm 0.078$ & $0.095 \pm 0.011$ & $0.200 \pm 0.005$ \\
3.20 & 179 & $2.428 \pm 0.153$ & $0.096 \pm 0.011$ & $0.190 \pm 0.006$ \\
3.40 & & &
\end{tabular}


Comparing the values of $A_{p p, h}$ and $A_{p p, f}$ it can be seen that the $V G R F / G$ generated during forefoot strike running reach higher values of mean amplitudes with smaller values of standard deviation. Moreover, the values of the approximated contact time $t_{c f, a}$ for forefoot strike running is smaller than the appropriate $t_{c h, a}$ for heel-strike running. It can also be seen that the impact peak during heel-strike running occurs on average about $41 \pm 0.007 \mathrm{~ms}$ after the contact of the heel with the ground.

Similar characteristic of the $V G R F / G$ curves can be found in (Nilsson \& Thorstensson, 1989), where values of the $V G R F / G$ amplitudes for heel strike and forefoot strike runners as a function of the speed of running are presented. In (Nilsson \& Thorstensson, 1989), the mean values of the VGRF amplitudes in the range of (2.10 to 2.70) $G$ and (2.30 to 2.75) $G$ are given for heel-strike and forefoot strike runners respectively in the range of speed of running 2.0-4.0 m/s. More significant differences between contact time of the foot with the ground $\left(t_{c h, a}, t_{c f, a}\right)$ presented in Table 2 and in Nilsson \& Thorstensson (1989) have been noticed. It is hard to indicate the reason for this due to the lack of detailed information about $t_{c}$ measurement technique in Nilsson \& Thorstensson (1989). The values presented in Tables 1 and 2 are consistent with the values presented in Wheeler (1982), where $t_{c} \approx 0.32 \mathrm{~s}$ for $f_{r}=2.4 \mathrm{~Hz}$ and $t_{c} \approx 0.17 \mathrm{~s}$ for $f_{r}=3.4 \mathrm{~Hz}$ can be found. In Wheeler (1982), the values of $t_{c}$ are defined without distinction between heel-strike and forefoot strike running technique. To the best of the author's knowledge, the data presented in the Tables 1 and 2, concerning the time of occurrence of the $V G R F$ curve indicators, are rarely analysed, and published in the studies like the one dedicated to the VGRF generated by people running.

\section{The VGRF models for forefoot and heel strike patterns}

\subsection{Forefoot strike pattern}

The VGRF generated during running is a near-periodic force with a time course similar to the half-cycle of a sine wave (especially in the case of the forefoot strike running). This force can be determined using Eqs. (1) - Fourier series or (2) - half-sine model presented in Bachmann et al. (1995) and Occhiuzzi et al. (2008), respectively.

$$
\frac{F(t)}{G}=1+\sum_{i=1}^{n} \alpha_{i} \sin \left(2 \pi i f_{r} t-\varphi_{i}\right)
$$




$$
\frac{F(t)}{G}=\left\{\begin{array}{c}
A_{r} \sin \left(\frac{\pi f_{r}}{k} t\right) \text { for } \quad j T_{r}<t \leq(j+k) T_{r} \\
0 \text { for } \quad(j+k) T_{r}<t \leq(j+1) T_{r},
\end{array}\right.
$$

where

$$
\begin{aligned}
k & =\frac{t_{c}}{T_{r}}, \\
A_{r} & =\frac{\pi}{2 k} .
\end{aligned}
$$

According to the recommendations presented in Bachmann et al. (1995), the values of the dynamic load factors (Fourier coefficients) $\alpha_{i}$ and the phase shifts $\varphi_{i}$ for the first three harmonics (three harmonics are usually assumed for modelling the VGRF) are $\alpha_{1}=1.6$, $\alpha_{2}=0.7, \alpha_{3}=0.2$, respectively; $\varphi_{i}$ - lack of recommendations (assumed $\varphi_{2}=\varphi_{3}=0$ ). In case of Eq. (2) in Occhiuzzi et al. (2008), $t_{c}=0.5 T_{r}$ is suggested for an ordinary running activity.

In Figure 6, exemplary charts of the $V G R F / G$ determined using Eqs. (1) and (2) (solid line) with the input parameters recommended in Bachmann et al. (1995) and Occhiuzzi et al. (2008) concerning the $V G R F / G$ acquired during laboratory tests (dashed line) are presented.

When using the input parameters recommended in Bachmann et al. (1995) and Occhiuzzi et al. (2008), there is significant inaccuracy in the estimation of the $V G R F / G$ values. The amplitudes of the $V G R F / G$ are overestimated ( $V G R F / G \approx 3.0$ occurs sporadically, e.g. during the sprint). Moreover, the simplified assumption of constantan values of the Fourier coefficients $\alpha_{i}$ leads to a constant $V G R F / G$ amplitude for each analysed running frequency. Simultaneously, Eq. (1) allows accurate estimation of $t_{c}$ value, but the assumption of $t_{c}=0.5 T_{r}$ in Eq. (2) according to Occhiuzzi et al. (2008) leads to incorrect estimation of $t_{c}$ and, consequently, incorrect estimation of the $V G R F / G$ amplitude (compare Eqs. (3) and (4)). The VGRF curves can be numerically compared using the mean value of normalised force $F_{a v}$ determined as the quotient of the area under the normalised $V G R F / G$ graph (the normalised impulse of force $J_{G}$ ) and contact time $t_{c}$ (duration of force) (Table 3 ).

In Table 3, normalised impulses determined for the forces acquired during laboratory tests are like the impulses determined for forces calculated using Eq. (2). Nevertheless, the average value of the normalised force determined for forces resulting from laboratory tests has the lowest value. The value of the normalised force determined for the forces resulting from Eq. (2) is the largest (substantially higher than in case of laboratory tests). Consequently, the dynamic response of the structure determined to consider the force described by Eq. (2) will be significantly overestimated (especially for the structures with low 

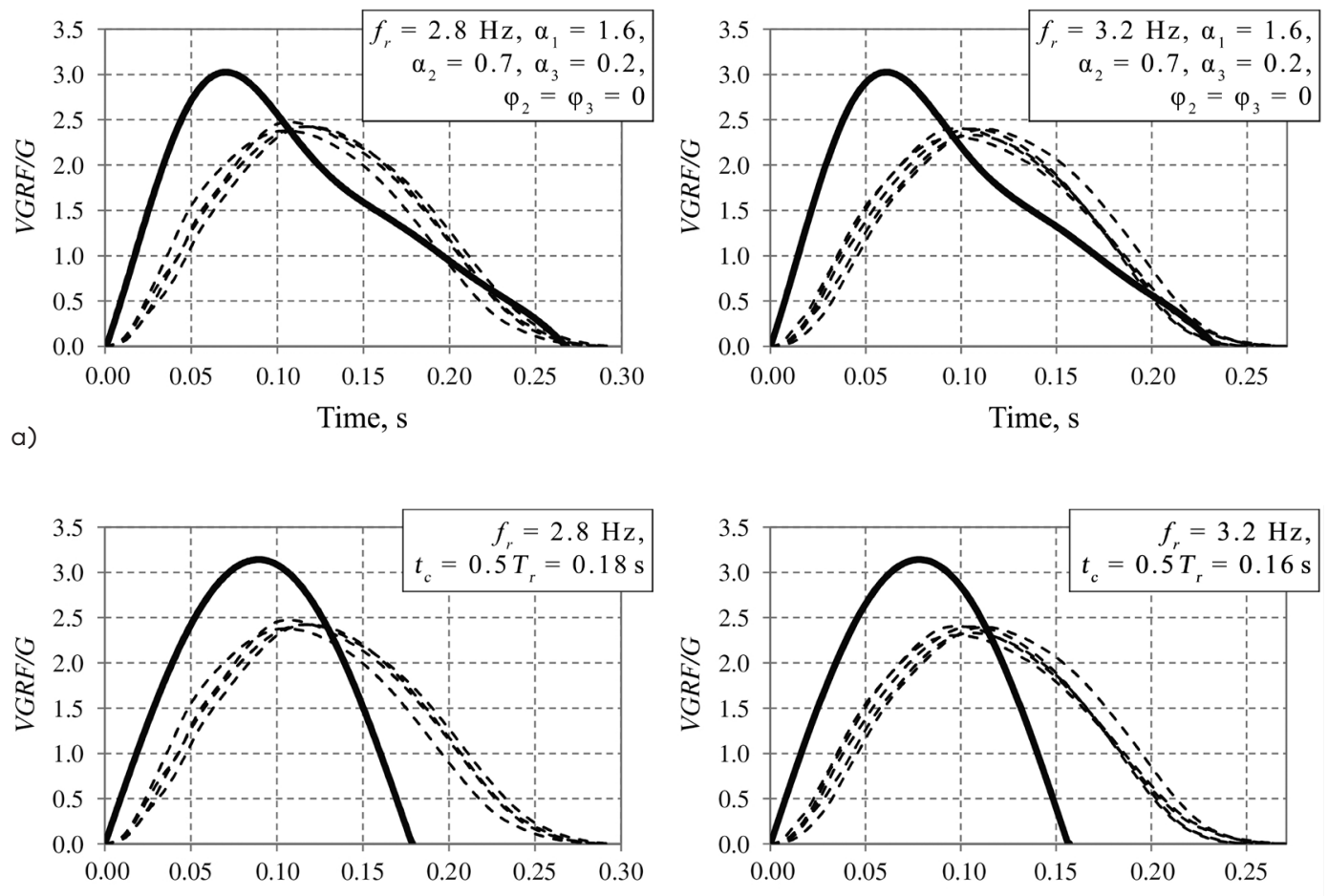

b)

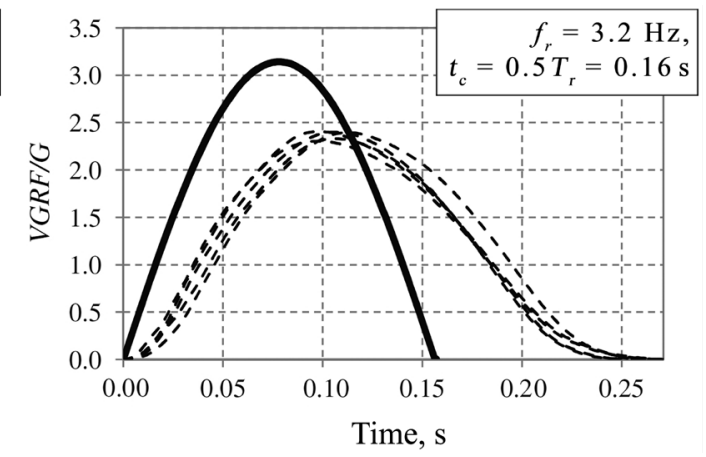

Figure 6. The normalised VGRF/G determined for $f_{r}=2.8 \mathrm{~Hz}$ and $f_{r}=3.2 \mathrm{~Hz}$ using: a) Eq. (1) for the input parameters by Bachmann et al. (1995), b) Eq. (2) for the input parameters by Occhiuzzi et al. (2008) (input parameters presented in the charts)

Table 3. Parameters of the VGRF/G curves presented in Figure 6

\begin{tabular}{|c|c|c|c|c|}
\hline $\begin{array}{l}\text { Frequency } \\
\qquad f_{r,} \mathrm{~Hz}\end{array}$ & VGRF/G curve & $\begin{array}{l}\text { Normalised } \\
\text { impulse } J_{G}, s\end{array}$ & $\begin{array}{c}\text { Contact time } \\
\qquad t_{c, s}\end{array}$ & $\begin{array}{c}\text { Normalised force } \\
\text { (mean value) } \\
F_{a v}=J_{G} / t_{c}\end{array}$ \\
\hline & Laboratory tests & 0.357 & 0.291 & 1.227 \\
\hline \multirow[t]{3}{*}{2.80} & Fig. 6a - Eq. (1) & 0.414 & 0.269 & 1.539 \\
\hline & Fig. 6 b - Eq. (2) & 0.357 & 0.178 & 2.006 \\
\hline & Laboratory tests & 0.318 & 0.262 & 1.214 \\
\hline \multirow[t]{2}{*}{3.20} & Fig. 6a - Eq. (1) & 0.362 & 0.235 & 1.540 \\
\hline & Fig. $6 b-$ Eq. (2) & 0.312 & 0.156 & 2.003 \\
\hline
\end{tabular}


damping). The case of forces determined using Eq. (1) is an intermediate case, which also leads to an overestimation of the vibration acceleration but a lesser extent than in the case of forces resulting from Eq. (2), mostly due to the lower mean value of the normalised force and different time course of the force.

It should be noted that Eqs. (1) and (2) allow estimating the $V G R F / G$ generated during forefoot running characterised by a lack of impact peak, but input parameters $\alpha_{i}$ and $t_{c}$ should be adjusted to improve the estimation of the $V G R F$.

More accurate estimation of the $V G R F / G$ by Eq. (1) can be achieved using the recommendations given in (Rainer \& Pernica, 1986; Rainer, Pernica, \& Allen, 1988). In the current analyses, the mean values of $\alpha_{i}$ established using recommendations given in (Rainer \& Pernica, 1986; Rainer et al., 1988) for the frequency range $2.4-3.4 \mathrm{~Hz}$ equal to $\alpha_{1}=1.3$, $\alpha_{2}=0.3, \alpha_{3}=0.1, \varphi_{2}=\varphi_{3}=0$ were used. The $V G R F / G$ curves determined using the corrected input parameters are presented in Figure 7 (normalised impulse $J_{G}=0.376 \mathrm{~s}$, contact time $t_{c}=0.280 \mathrm{~s}$, normalised force $F_{a v}=1.343$ for $f_{r}=2.80 \mathrm{~Hz}$ and $J_{G}=0.329 \mathrm{~s}, t_{c}=0.244 \mathrm{~s}, F_{a v}=1.348$ for $f_{r}=3.20 \mathrm{~Hz}$ (compare Table 3)).

The input parameters of Eq. (1) were analysed and corrected once again to better reconcile the VGRF/G curve with the results of laboratory tests. The analyses revealed that $\alpha_{i}$ values might be assumed in the range of $\alpha_{1}=1.0-1.7, \alpha_{2}=0.0-0.3, \alpha_{3}=0.0-0.1$ and the phase shifts values $\varphi_{2}=\varphi_{3}=0$ should be left unchanged. In Figure 8, the $V G R F / G$ determined using adjusted $\alpha_{i}$ values are presented (normalised impulse $J_{G}=0.384 \mathrm{~s}$, contact time $t_{c}=0.263 \mathrm{~s}$, the normalised force $F_{a v}=1.460$ for $f_{r}=2.80 \mathrm{~Hz}$
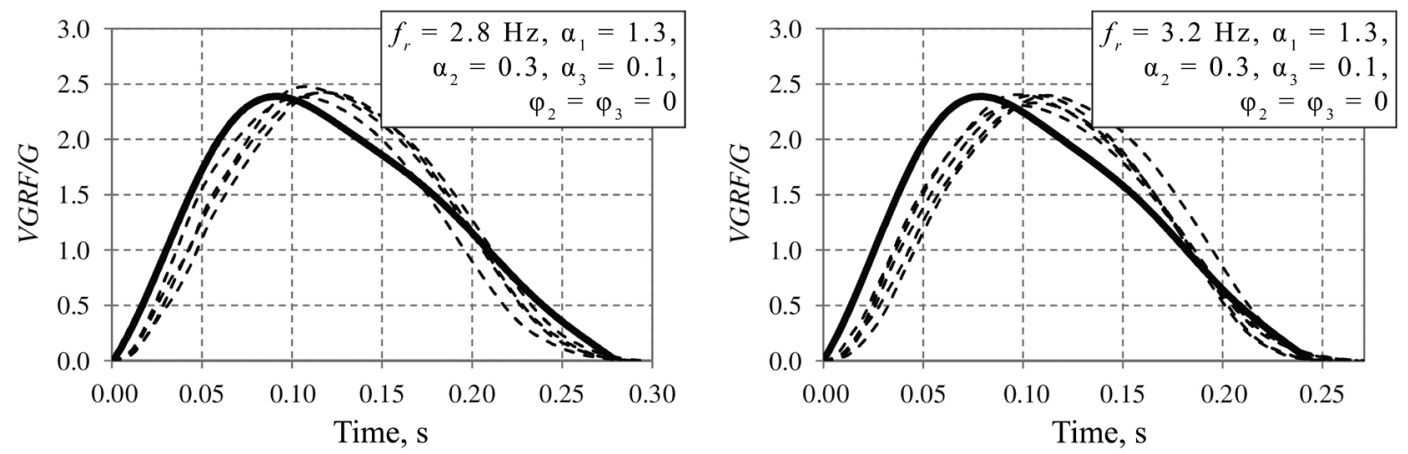

Figure 7. The normalised VGRF/G determined for $f_{r}=2.8 \mathrm{~Hz}$ and $f_{r}=3.2 \mathrm{~Hz}$ using Eq. (1) for input parameters recommended in Rainer \& Pernica (1986) and Rainer et al. (1988) (input parameters presented in the charts) 

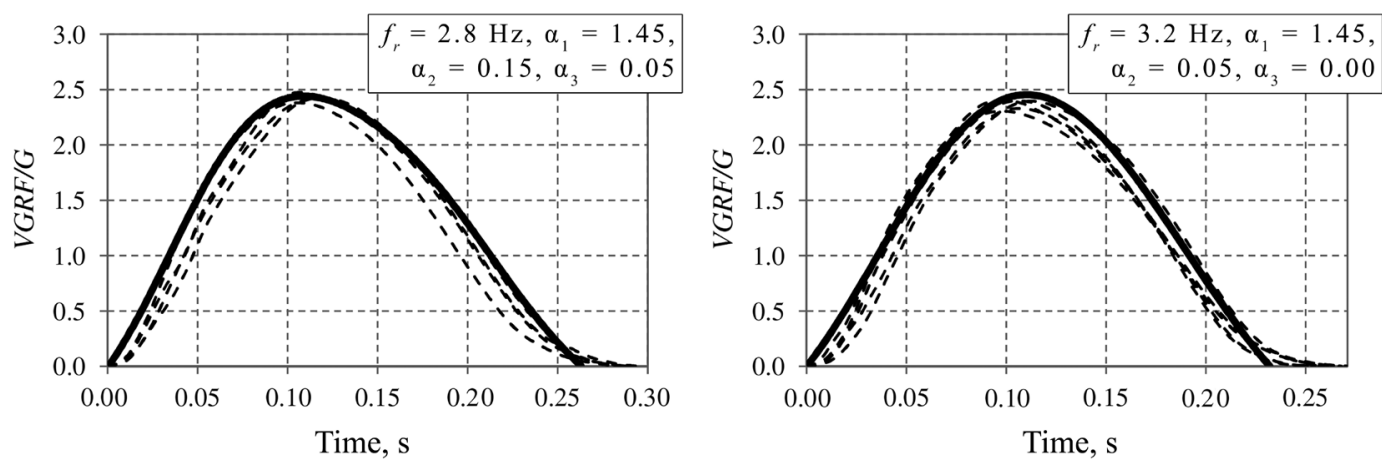

Figure 8. The normalised VGRF/G determined for $f_{r}=2.8 \mathrm{~Hz}$ and $f_{r}=3.2 \mathrm{~Hz}$ using Eq. (1) for adjusted input parameters (input parameters presented in the charts)

and $J_{G}=0.336 \mathrm{~s}, t_{c}=0.232 \mathrm{~s}, F_{a v}=1.448$ for $f_{r}=3.20 \mathrm{~Hz}$ (compare Table 3)).

It is worth noting that the values of $\alpha_{1}=1.0-1.3$ and $\alpha_{2}=0.2-0.3$ are appropriate to determine the $V G R F / G$ generated during a slow pace of running $\left(f_{r} \leq 2.6 \mathrm{~Hz}\right)$, whereas $\alpha_{1}=1.4-1.7$ and $\alpha_{2}=0.0-0.20$ are appropriate to determine the $V G R F / G$ generated during normal and fast pace of running $\left(f_{r}>2.6 \mathrm{~Hz}\right)$. Moreover, it is worth noting that the changes in the value of $\alpha_{3}$ (assumed in the range of 0.0-0.1) allow for a slight correction of the $V G R F / G$ values, but have no significant impact on the $V G R F / G$ course.

In case of determination of the $V G R F / G$ using Eq. (2), it is worth noting that the amplitude of the $V G R F / G$ (described by Eq. (4)) is
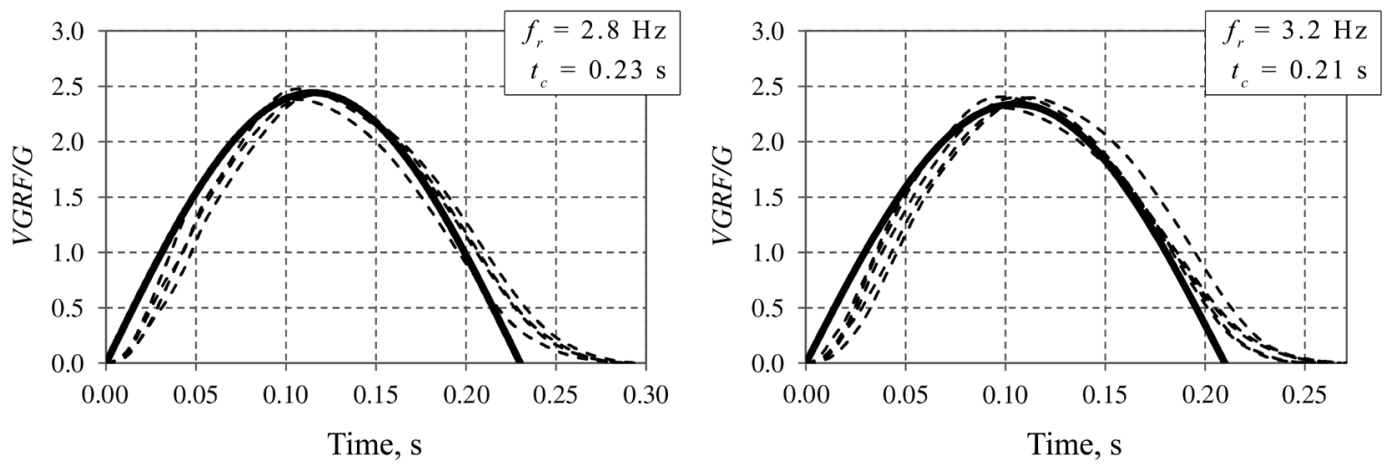

Figure 9. The normalised VGRF/G determined for $f_{r}=2.8 \mathrm{~Hz}$ and $f_{r}=3.2 \mathrm{~Hz}$ using Eq. (2) using adjusted $t_{c, f}$ values (input parameters presented in the charts) 
inversely proportional to the value of the contact time of the foot with the ground $t_{c}$. The analyses revealed that the amplitude of the $V G R F / G$ is very sensitive to the value of $t_{c}$. The assumption of the underestimated $t_{c}$, e.g. $t_{c}=0.5 T_{r}$, leads to a highly overestimated amplitude of the $V G R F / G$ (Figure 6). The correct mean value of $t_{c}$ for the case of forefoot strike running $\left(t_{c}=t_{c, f}\right)$ can be estimated using Eq. (5) developed for the case of forefoot strike running, considering the approximated contact time values $t_{c f, a}$ presented in Table 2 .

$$
t_{c, f}=0.166+5.25 \mathrm{e}^{-1.58 f_{r}} .
$$

In Figure 9, the $V G R F / G$ determined using Eq. (2) using adjusted $t_{c, f}$ values are presented $\left(J_{G}=0.357 \mathrm{~s}, t_{c}=0.23 \mathrm{~s}, F_{a v}=1.552\right.$ for $f_{r}=$ $2.80 \mathrm{~Hz}$ and $J_{G}=0.313 \mathrm{~s}, t_{c}=0.21 \mathrm{~s}, F_{a v}=1.490$ for $f_{r}=3.20 \mathrm{~Hz}$ (compare Table 3)).

\subsection{Heel-strike pattern}

To model the $V G R F / G$ generated by heel-strike runners, i.e. with the $V G R F / G$ containing an additional impact peak), a new supplementary function of impact peak $\varphi_{i p}(t)$ was defined in the form of Eq. (6) (author's proposal).

$$
\frac{\varphi_{i p}(t)}{G}=A_{\varphi} \sin \left(\alpha_{\varphi} \pi f_{r} t\right)^{\beta_{\varphi}} .
$$

In Figure 10, the illustrations of $\varphi_{i p}(t) / G$ with the $V G R F / G$ determined using Eqs. (1) and (2) in relation to the results of laboratory tests (dashed lines) are presented.
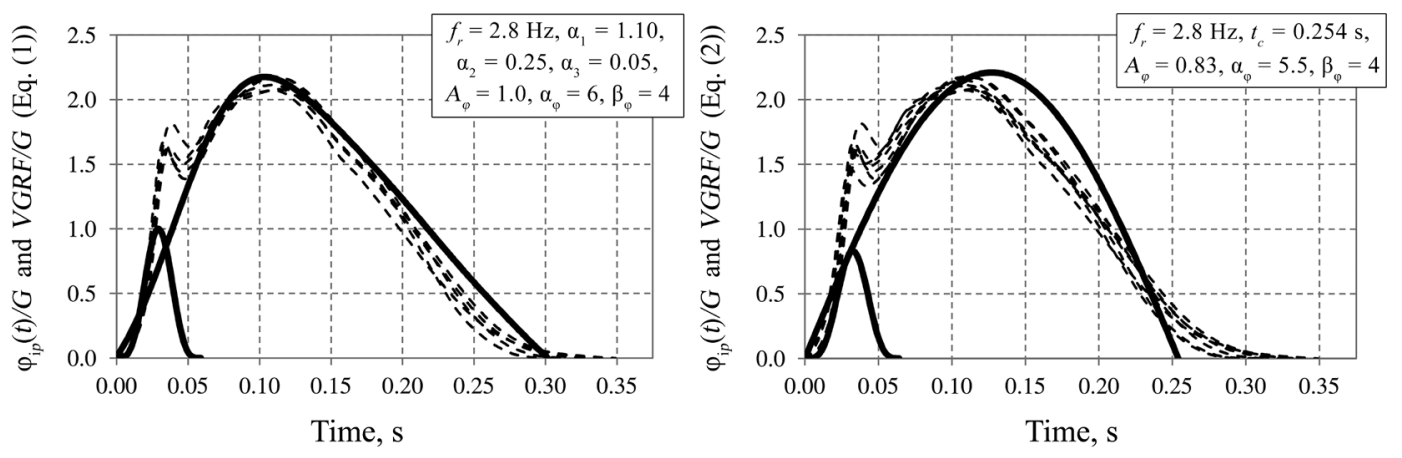

Figure 10. Illustration of the normalised impact peak $\varphi_{i p}(t) / G$ with the VGRF/G determined using Eqs. (1) and (2) and the results of laboratory tests (dashed lines) (input parameters presented in the charts) 
In order to calculate the $V G R F / G$ generated by heel-strike runners, the impact peak function should be added (in the initial time period $0 \leq t \leq \lambda T_{r}$, where $\lambda=0.17$ ) to the $V G R F / G$ determined using Eq. (1) or (2). Eq. (7) is proposed by the author of this paper.

$$
\frac{\Phi(t)}{G}=\left\{\begin{array}{c}
\frac{F(t)}{G}+\frac{\varphi_{i p}(t)}{G} \text { for } 0<t \leq \lambda T_{r} ; \\
\frac{F(t)}{G} \text { for } \lambda T_{r}<t \leq t_{c} .
\end{array}\right.
$$

In the proposed method, Eq. (1) or (2) were used as a primary $V G R F / G$ function. For the correct estimation of the $V G R F / G$ using Eq. (2), the appropriate value of the contact time of the foot with the ground $t_{c}=t_{c, h}$ for the case of heel-strike running must be used. The mean value of $t_{c, h}$ can be estimated using Eq. (8) formulated by the author for the case of heel-strike running, taking into account the approximated contact time values $t_{c h, a}$ presented in Table 1.

$$
t_{c, h}=0.215+30 \mathrm{e}^{-2.35 f_{r}} .
$$

Furthermore, due to the different slope of the curve generated using basic Eq. (1) or (2), in the initial period (Figure 6), $A_{\varphi}$ value should be adopted adequately to the chosen basic equation. A typical $A_{\varphi}$ value can be assumed to be in the range of $0.5-1.3\left(A_{\varphi} \geq 1.0\right.$ applies to the $V G R F / G$ calculated using Eq. (1)). The value of the impact peak location coefficient $\alpha_{\varphi}$ should be assumed to be in the range $\alpha_{\varphi}=4-8$ $\left(\alpha_{\varphi}\right.$ can be a decimal number). For $\alpha_{\varphi}=5-8$, the impact peak on $V G R F / G$ graph will occur at the time of $t_{i p}=30-50 \mathrm{~ms}$ after contact of the foot with the ground in frequency range $f_{r}=2.4-2.8 \mathrm{~Hz}$. For frequency range $f_{r}=2.8-3.4, \alpha_{\varphi}=4-6$ can be assumed. The coefficient should be adequately adjusted to the running frequency to achieve the location of the impact peak at $t_{i p}=30-45 \mathrm{~ms}$ after contact of the foot with the ground. $\beta_{\varphi}=2,3,4,5, \ldots$ can be assumed. The recommended value is $\beta_{\varphi}=4$ ( $\beta_{\varphi}$ can be a decimal number).

In Figure 11, the normalised $\Phi(t) / G$ graphs with the results of laboratory tests are presented.

Another way of modelling the $V G R F / G$ generated by people running was proposed by Racic \& Morin (2014). In this proposal, the $V G R F / G$ curve was modelled as the sum of the Gaussian functions. Using a procedure like that proposed by Racic \& Morin (2014), the Gaussian functions for heel-strike runners were determined. In the analyses, the form of the Gaussian function defined by Eq. (9) was assumed; this 

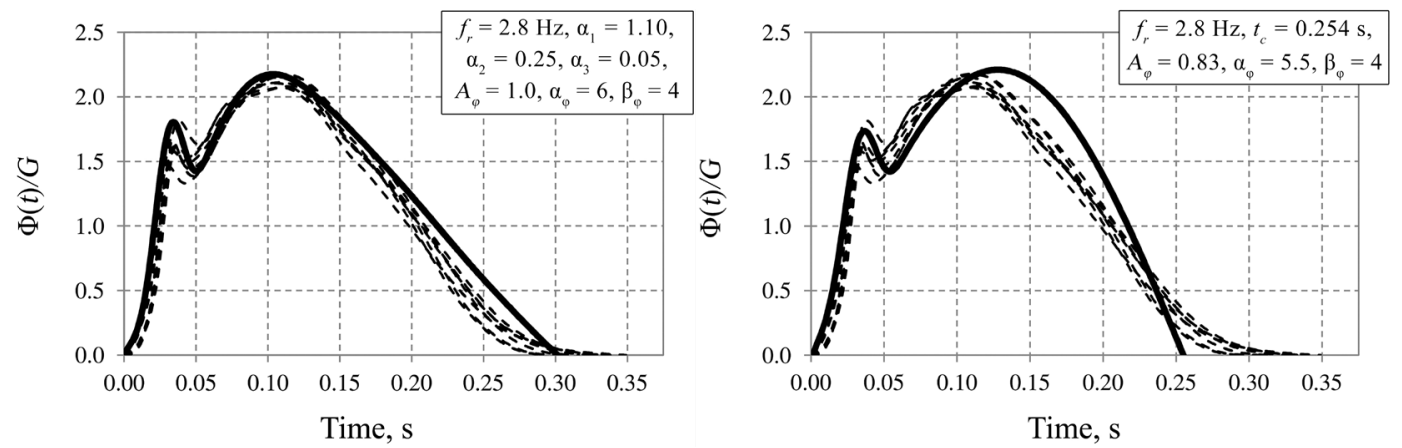

Figure 11. Illustration of the normalised $\Phi(t) / G$ functions with the results of laboratory tests (input parameters presented in the charts)

is slightly different from the proposition presented in Racic \& Morin (2014).

$$
\Omega(t)=\sum_{p=1}^{5} A_{p} \mathrm{e}^{\left[-\ln 2 \frac{\left(t-t_{p}\right)^{2}}{\sigma_{p}^{2}}\right]} .
$$

Furthermore, the sum of five components of the Gaussian function was accepted instead of the four components used by Racic \& Morin (2014). Acceptance allowed precisely fitting the artificially generated $V G R F / G$ curve to the $V G R F$ generated by the heel-strike runners containing a clearly a visible impact peak (Figures 5 and 11).

In contrast to proposals of Racic \& Morin (2014), instead of one $V G R F / G$ template, the set of $V G R F / G$ templates was defined for running frequencies $2.40 \mathrm{~Hz}, 2.60 \mathrm{~Hz}, 2.80 \mathrm{~Hz}, 3.00 \mathrm{~Hz}, 3.20 \mathrm{~Hz}, 3.40 \mathrm{~Hz}$ (frequency increment $0.2 \mathrm{~Hz}$ ). The $V G R F / G$ templates were determined as templates reaching the mean values of the $A_{p p, h}$ curve indicators (Figure $5 \mathrm{a}$ and Table 1) and matching the mean $V G R F / G$ trace arising from the analysed samples for each running frequency separately (Figure 12). The number of runners and the $V G R F / G$ samples taken into account for each running frequencies was: 7 runners (111 VGRF samples) for $f_{r}=2.40 \mathrm{~Hz}$; 6 runners (114 VGRF samples) for $f_{r}=2.60 \mathrm{~Hz} ; 6$ runners $(139 V G R F$ samples) for $f_{r}=2.80 \mathrm{~Hz}$; 6 runners $\left(132 V G R F\right.$ samples) for $f_{r}=3.00 \mathrm{~Hz}$; 5 runners (76 VGRF samples) for $f_{r}=3.20 \mathrm{~Hz} ; 4$ runners (80 VGRF samples) for $f_{r}=3.40 \mathrm{~Hz}$ (Table 1 ). The parameters of the elaborated templates are presented in Tables 4-9.

For the elaborated templates, analyses of the variability of the parameters of the Gaussian functions $A_{p}, t_{p}, \sigma_{p}$ (for $p=1,2, \ldots, 5$ ) 

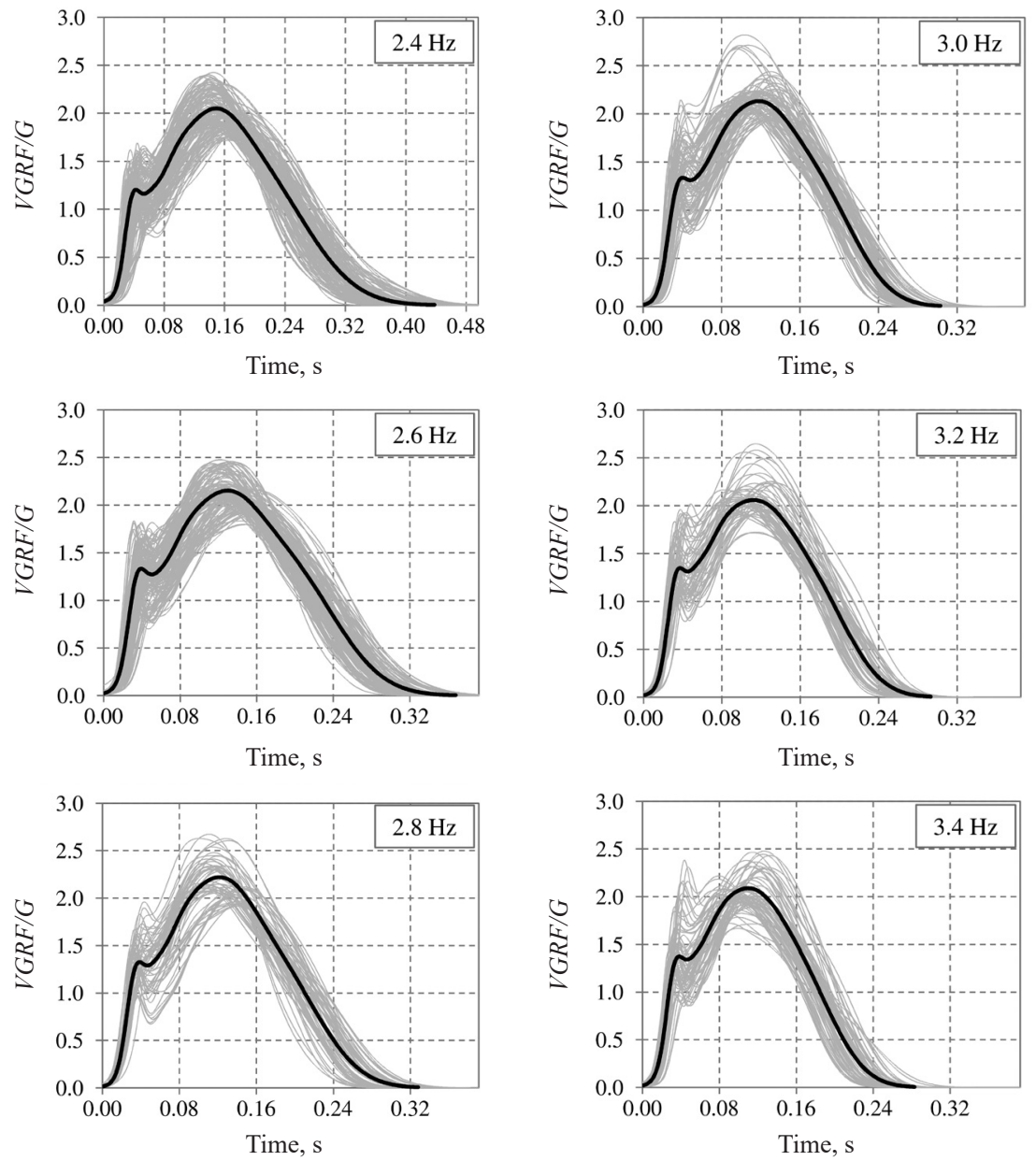

Figure 12. The templates (solid black line) determined for running frequencies of $f_{r}=2.4-3.4 \mathrm{~Hz}$ with exemplary laboratory tests results (thin grey lines)

considered as a function of the running frequency $f_{r}$ were performed. The obtained results are presented in Figure 13, where the trends in the distribution of the Gaussian function parameters can be observed. The trend lines presented in Figure 13 (solid line) were determined using $2^{\text {nd }}$-degree polynomial interpolation and presented in the form of Eqs. (10)-(12). 


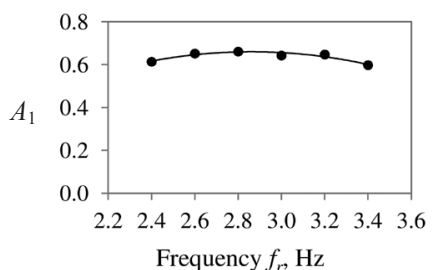

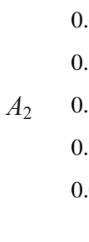

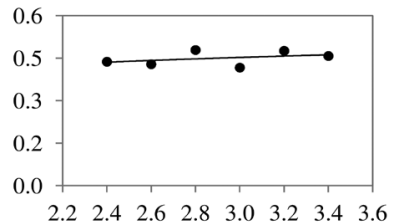

Running
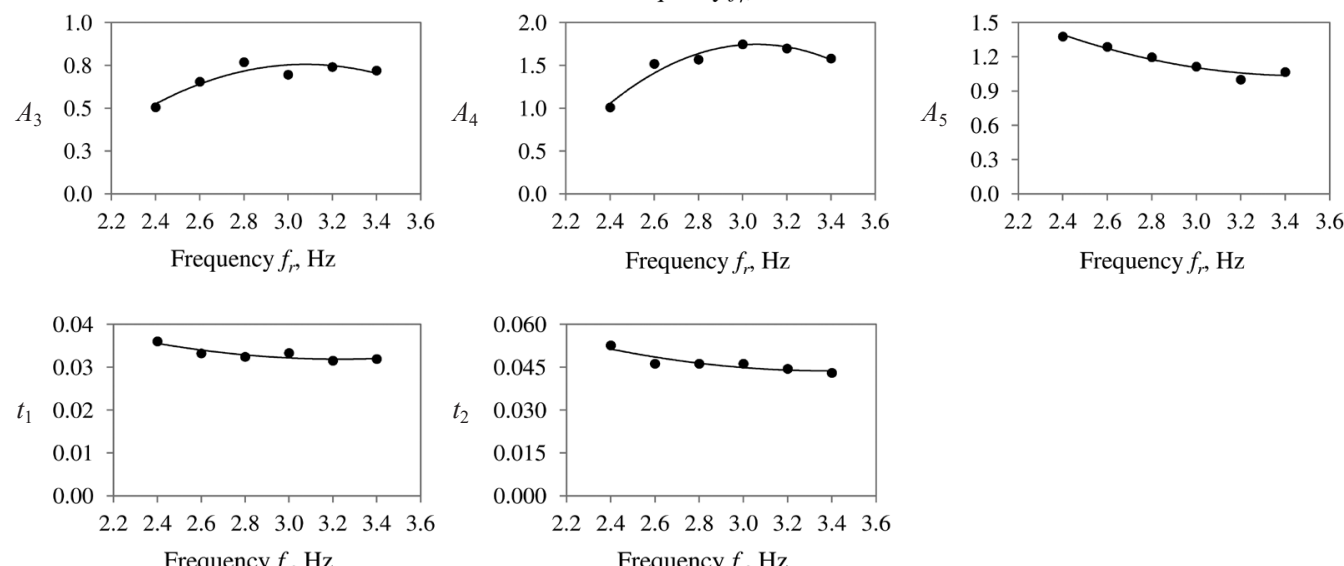

Frequency $f_{r}, \mathrm{~Hz}$
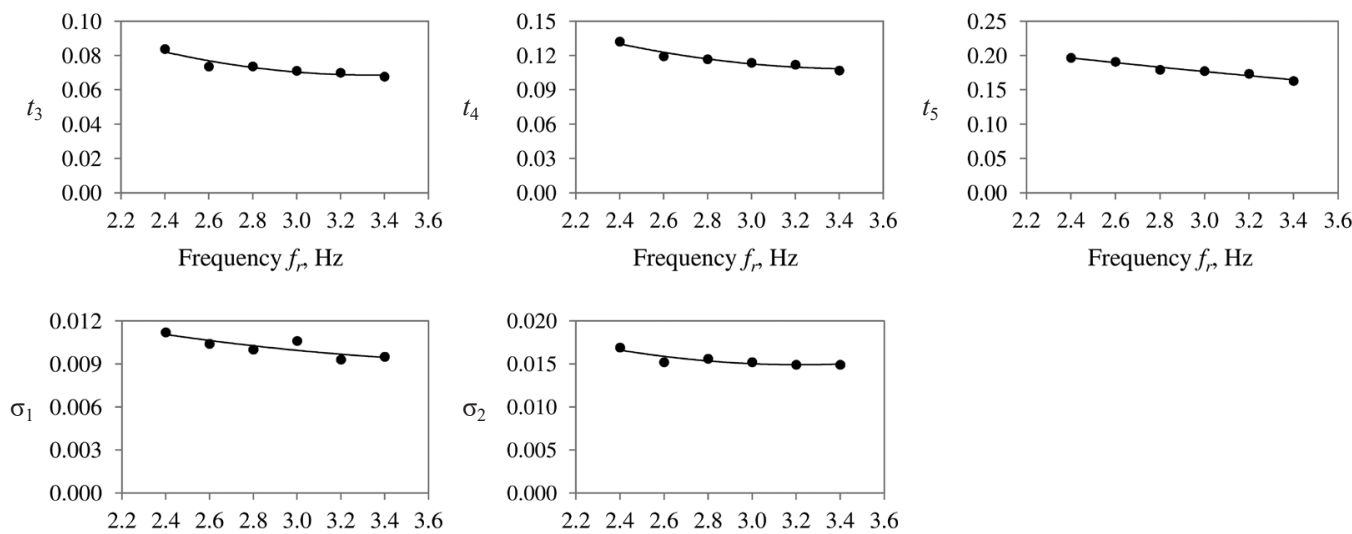

Frequency $f_{r}, \mathrm{~Hz}$

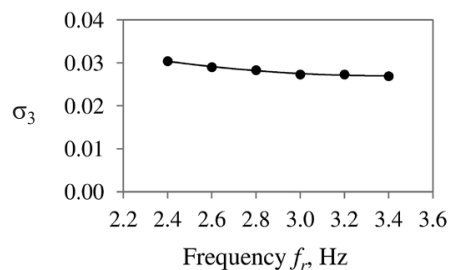

Frequency $f_{r}, \mathrm{~Hz}$
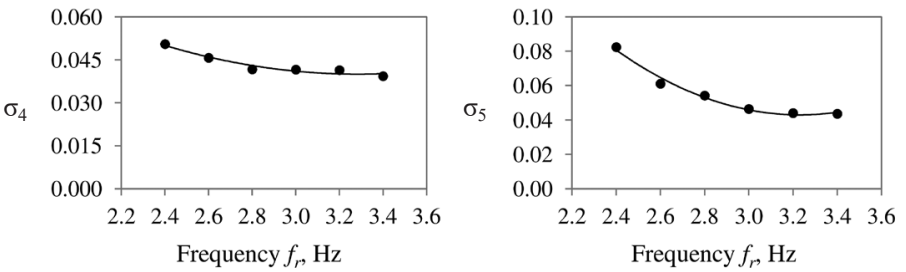

Figure 13. Variability of the Gaussian function parameters $A_{p 1} t_{p 1} \sigma_{p}$ considered as a function of the running frequency $f_{r}$ (black dots - parameters of templates presented in Tables 4-9; solid line - trend line determined using $2^{\text {nd }}$-degree polynomial interpolation) 
Table 4. Parameters of the template for running frequency of $2.4 \mathrm{~Hz}$

\begin{tabular}{|c|c|c|c|c|c|c|}
\hline \multirow{2}{*}{$\begin{array}{l}\text { Frequency } f_{r r} \\
\mathrm{~Hz}\end{array}$} & \multirow{2}{*}{ Parameters } & \multicolumn{5}{|c|}{ Parameters of the Gaussian function } \\
\hline & & $p=1$ & $p=2$ & $p=3$ & $p=4$ & $p=5$ \\
\hline \multirow{3}{*}{2.4} & $A_{p}$ & 0.6132 & 0.4375 & 0.5052 & 1.0090 & 1.3767 \\
\hline & $t_{p}$ & 0.0360 & 0.0526 & 0.0838 & 0.1322 & 0.1967 \\
\hline & $\sigma_{p}$ & 0.0112 & 0.0169 & 0.0304 & 0.0505 & 0.0824 \\
\hline
\end{tabular}

Table 5. Parameters of the template for running frequency of $2.6 \mathrm{~Hz}$

\begin{tabular}{ccccccc}
\hline \multirow{2}{*}{$\begin{array}{c}\text { Frequency } \boldsymbol{f}_{\boldsymbol{r} \boldsymbol{r}} \\
\mathbf{H z}\end{array}$} & Parameters & \multicolumn{5}{c}{ Parameters of the Gaussian function } \\
\cline { 3 - 7 } & & $\mathbf{p = 1}$ & $\mathbf{p = 2}$ & $\mathbf{p}=\mathbf{3}$ & $\mathbf{p = 4}$ & $\boldsymbol{p}=\mathbf{5}$ \\
\hline \multirow{2}{*}{2.6} & $A_{p}$ & 0.6514 & 0.4289 & 0.6545 & 1.5175 & 1.2871 \\
& $t_{p}$ & 0.0332 & 0.0462 & 0.0736 & 0.1193 & 0.1909 \\
& $\sigma_{p}$ & 0.0104 & 0.0152 & 0.0290 & 0.0457 & 0.0611 \\
\hline
\end{tabular}

Table 6. Parameters of the template for running frequency of $2.8 \mathrm{~Hz}$

\begin{tabular}{ccccccc}
\hline \multirow{2}{*}{$\begin{array}{c}\text { Frequency } \boldsymbol{f}_{\boldsymbol{r} \boldsymbol{r}} \\
\mathbf{H z}\end{array}$} & Parameters & \multicolumn{5}{c}{ Parameters of the Gaussian function } \\
\cline { 2 - 7 } & & $\mathbf{p = 1}$ & $\mathbf{p = 2}$ & $\mathbf{p}=\mathbf{3}$ & $\mathbf{p = 4}$ & $\mathbf{p = 5}$ \\
\hline \multirow{2}{*}{2.8} & $A_{p}$ & 0.6605 & 0.4790 & 0.7682 & 1.5671 & 1.1958 \\
& $t_{p}$ & 0.0324 & 0.0462 & 0.0737 & 0.1168 & 0.1793 \\
& $\sigma_{p}$ & 0.0100 & 0.0156 & 0.0283 & 0.0417 & 0.0542 \\
\hline
\end{tabular}

Table 7. Parameters of the template for running frequency of $3.0 \mathrm{~Hz}$

\begin{tabular}{ccccccc}
\hline \multirow{2}{*}{$\begin{array}{c}\text { Frequency } \boldsymbol{f}_{\boldsymbol{r} \boldsymbol{r}} \\
\mathbf{H z}\end{array}$} & Parameters & \multicolumn{5}{c}{ Parameters of the Gaussian function } \\
\cline { 3 - 7 } & & $\mathbf{p = 1}$ & $\mathbf{p = 2}$ & $\mathbf{p = 3}$ & $\mathbf{p = 4}$ & $\mathbf{p = 5}$ \\
\hline \multirow{3}{*}{3.0} & $A_{p}$ & 0.6422 & 0.4168 & 0.6953 & 1.7457 & 1.1136 \\
& $t_{p}$ & 0.0333 & 0.0462 & 0.0711 & 0.1137 & 0.1774 \\
& $\sigma_{p}$ & 0.0106 & 0.0152 & 0.0273 & 0.0416 & 0.0464 \\
\hline
\end{tabular}

Table 8. Parameters of the template for running frequency of $3.2 \mathrm{~Hz}$

\begin{tabular}{|c|c|c|c|c|c|c|}
\hline \multirow{2}{*}{$\begin{array}{l}\text { Frequency } f_{r \prime} \\
\qquad \mathrm{Hz}\end{array}$} & \multirow{2}{*}{ Parameters } & \multicolumn{5}{|c|}{ Parameters of the Gaussian function } \\
\hline & & $p=1$ & $p=2$ & $p=3$ & $p=4$ & $p=5$ \\
\hline \multirow{3}{*}{3.2} & $A_{p}$ & 0.6470 & 0.4760 & 0.7403 & 1.6968 & 0.9996 \\
\hline & $t_{p}$ & 0.0315 & 0.0444 & 0.0700 & 0.112 & 0.1735 \\
\hline & $\sigma_{p}$ & 0.0093 & 0.0149 & 0.0273 & 0.0414 & 0.0440 \\
\hline
\end{tabular}


Table 9. Parameters of the template for running frequency of $3.4 \mathrm{~Hz}$

\begin{tabular}{ccccccc}
\hline \multirow{2}{*}{$\begin{array}{c}\text { Frequency } \\
\mathbf{H z}\end{array}$} & Parameters & \multicolumn{5}{c}{ Parameters of the Gaussian function } \\
\cline { 2 - 6 } & & $\mathbf{p = 1}$ & $\mathbf{p}=\mathbf{2}$ & $\mathbf{p}=\mathbf{3}$ & $\mathbf{p = 4}$ & $\mathbf{p = 5}$ \\
\hline \multirow{3}{*}{3.4} & $A_{p}$ & 0.5975 & 0.4579 & 0.7195 & 1.5795 & 1.0661 \\
& $t_{p}$ & 0.0319 & 0.0430 & 0.0677 & 0.1069 & 0.1630 \\
& $\sigma_{p}$ & 0.0095 & 0.0149 & 0.0269 & 0.0393 & 0.0436 \\
\hline
\end{tabular}

The amplitude of the Gaussian curve $A_{p}$ :

$$
\begin{aligned}
& A_{1}=-0.2034 f_{r}^{2}+1.1642 f_{r}-1.0063, \\
& A_{2}=-0.005 f_{r}^{2}+0.0546 f_{r}+0.3332, \\
& A_{3}=-0.5024 f_{r}^{2}+3.0932 f_{r}-4.0061, \\
& A_{4}=-1.5728 f_{r}^{2}+9.6319 f_{r}-13.0028, \\
& A_{5}=0.3079 f_{r}^{2}-2.1426 f_{r}+4.7614 .
\end{aligned}
$$

Position of the peak of the Gaussian curve $t_{p}$ :

$$
\begin{aligned}
& t_{1}=0.0054 f_{r}^{2}-0.0346 f_{r}+0.0877, \\
& t_{2}=0.0079 f_{r}^{2}-0.0537 f_{r}+0.1345, \\
& t_{3}=0.0155 f_{r}^{2}-0.1033 f_{r}+0.2407, \\
& t_{4}=0.0188 f_{r}^{2}-0.1309 f_{r}+0.3358, \\
& t_{5}=0.0033 f_{r}^{2}-0.0507 f_{r}+0.2994 .
\end{aligned}
$$

The width of the Gaussian curve $\sigma_{p}$ :

$$
\begin{aligned}
& \sigma_{1}=0.0006 f_{r}^{2}-0.0052 f_{r}+0.02, \\
& \sigma_{2}=0.0025 f_{r}^{2}-0.0164 f_{r}+0.0412, \\
& \sigma_{3}=0.0035 f_{r}^{2}-0.0236 f_{r}+0.0669, \\
& \sigma_{4}=0.0128 f_{r}^{2}-0.0842 f_{r}+0.1782, \\
& \sigma_{5}=0.0547 f_{r}^{2}-0.3533 f_{r}+0.6137 .
\end{aligned}
$$

The presented variability of the Gaussian function parameters in function of the frequency of running complement and extend the result of analyses presented in Racic \& Morin (2014). Knowledge of the variability of function parameters in function of the frequency of running allows easily (manually) creating the $V G R F$ curves. The presented approach allows simplifying the way of modelling of the $V G R F$ 
curves presented in Racic \& Morin (2014), which the authors called "too complex to generate artificial forces manually".

In Figures 14 and 15, collections of the $V G R F / G$ templates determined using the data from Tables 4-9 (original data before interpolation) and templates determined using the interpolated data (data described by Eqs. (10)-(12)) are presented. Additionally, in Figure 15, the coefficient of determination $R^{2}$ calculated using the original and interpolated templates as well as the Gaussian curves representing five components of Eq. (9) (dashed lines) are shown. High values of $R^{2}$ coefficients confirm the very good fit of the interpolated templates with the original data.

Analysing Figure 13 and the Gaussian curves presented in Figure 15 (dashed lines), some general trends can be observed. Specifically, there is a small variability of the values of amplitudes $A_{1}$ and $A_{2}$, a definite increase in the values of amplitude $A_{4}$ (to a lesser extent also $A_{3}$ ) in the frequency range $2.4-3.0 \mathrm{~Hz}$, a definite and permanent decrease in the value of amplitude $A_{5}$ and a permanent decrease in the values of $t_{p}$ and $\sigma_{p}$ for $p=1-5$. Furthermore, in Figure 13 it can be observed that the changes in the values of amplitude $A_{4}$ and the Gaussian function width parameter $\sigma_{5}$ are more rapid than the changes in other parameters of the Gaussian functions. In addition, for $f_{r}=2.4 \mathrm{~Hz}$ (to a lesser extent also for $f_{r}=2.6 \mathrm{~Hz}$ ), a relatively large width of the Gaussian curve (i.e. $\sigma_{5}$ value) is observed (see Table 4 and Figure 15). The peak amplitude of the $V G R F / G$ template for $f_{r}=2.4 \mathrm{~Hz}$ (slow running) depends to a large extent on amplitudes $A_{4}$ and $A_{5}$. The width of the Gaussian curves decreases as the running frequency increases. For $f_{r}>2.6 \mathrm{~Hz}$, the peak amplitude of the $V G R F / G$ template depends mainly on the value of amplitude $A_{4}$.
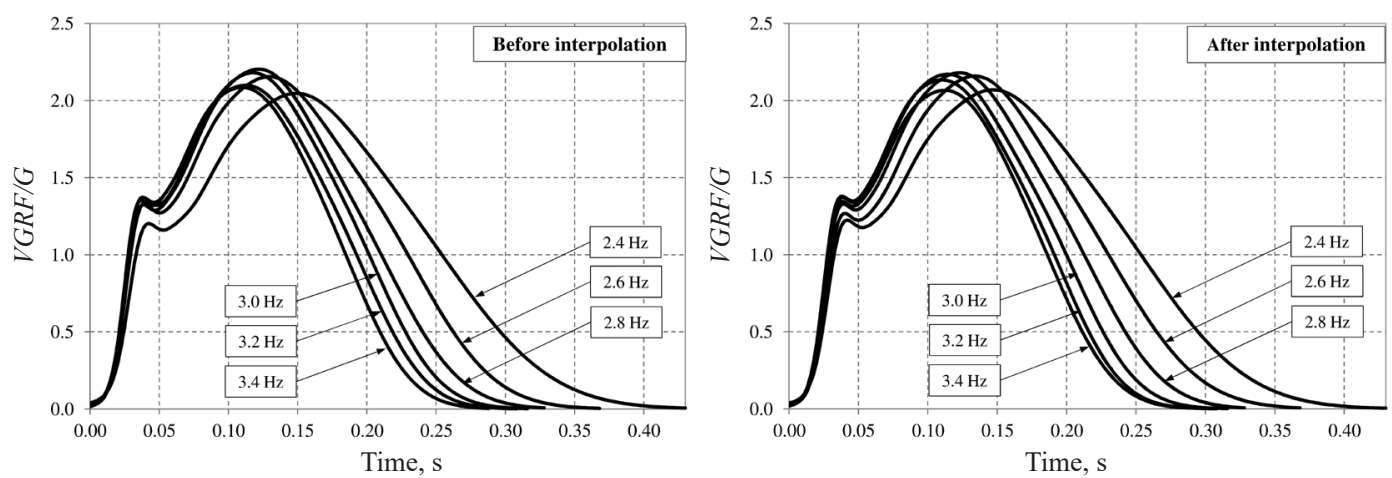

Figure 14. Collection of the VGRF/G templates in frequency range $f_{r}=2.4-3.4 \mathrm{~Hz}$ before and after interpolation of the parameters of the Gaussian functions 
It should be emphasised that defined $V G R F / G$ templates represent the mean values of the normalised ground reaction forces $V G R F / G$. In case of modelling the $V G R F / G$ generated by a randomly selected person, the $V G R F / G$ templates should be used with caution because of the high sensitivity of the $V G R F / G$ values determined through the Gaussian functions to changes of $t_{p}$ and $\sigma_{p}$ parameters. In practice, modelling of the changes of the $V G R F / G$ values can be realised by modifications of $A_{p}$ values enabling reaching of the $V G R F / G$ amplitudes presented in Table 1 for heel-strike pattern runners or in Table 2 for forefoot pattern runners (assuming $A_{1}=0$ for forefoot pattern runners), leaving $t_{p}$ and $\sigma_{p}$ values unchanged or with only minor changes (Tables 11 and 12).
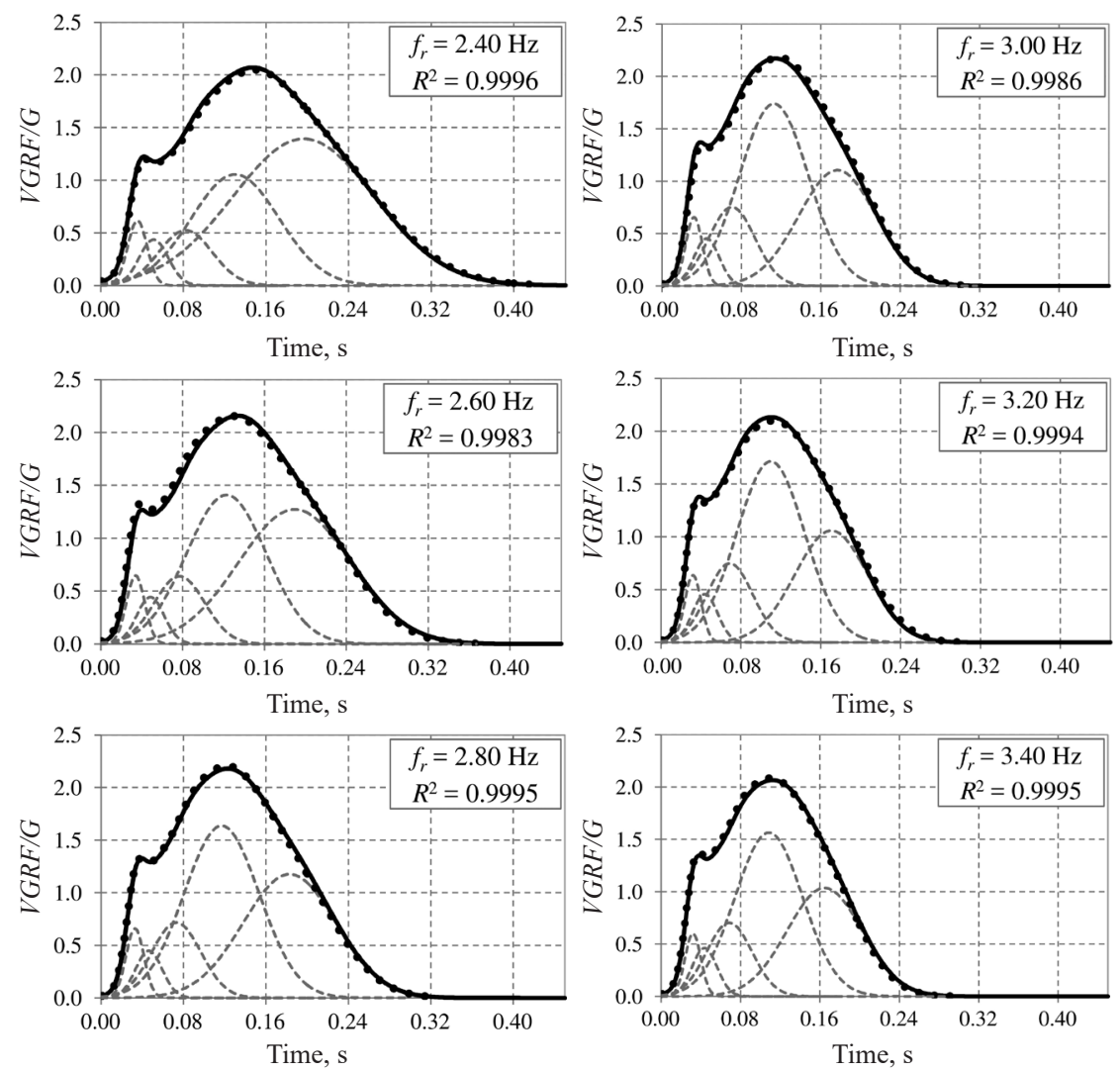

Figure 15. Comparisons of the VGRF/G templates before and after interpolation of parameters of the Gaussian function in frequency range $f_{r}=2.4-3.4 \mathrm{~Hz}$ : black dotted line - the VGRF/G templates before interpolation of parameters of the Gaussian function; solid black line the VGRF/G templates after interpolation of parameters of the Gaussian function, grey dashed line - the Gaussian curves (five components of Eq. (9)) 
In Tables 10, 11 and 12, examples of the mean values and the values of $95 \%$ confidence intervals (CI) of the Gaussian function parameters $A_{p}$, $t_{p}$, and $\sigma_{p}$ determined based on laboratory tests for one running person (heel-strike pattern runner) are presented.

Table 10. Examples of $A_{p}$ mean values and $95 \%$ confidence intervals $(\mathrm{Cl})$ determined for one heel-strike pattern runner in frequency range $f_{r}=2.4-3.4 \mathrm{~Hz}$ (results of laboratory tests)

\begin{tabular}{|c|c|c|c|c|c|}
\hline \multirow{2}{*}{$\begin{array}{c}\text { Frequency } f_{r \prime} \\
\mathrm{Hz}\end{array}$} & \multicolumn{5}{|c|}{ Amplitudes of the Gaussian functions $A_{p}$} \\
\hline & $A_{1} \pm \mathrm{Cl}$ & $A_{2} \pm \mathrm{Cl}$ & $A_{3} \pm \mathrm{Cl}$ & $A_{4} \pm \mathrm{Cl}$ & $A_{5} \pm \mathrm{Cl}$ \\
\hline \multirow{2}{*}{2.4} & $0.7909 \pm$ & $0.3891 \pm$ & $0.5485 \pm$ & $0.8602 \pm$ & $1.4617 \pm$ \\
\hline & 0.0435 & 0.0353 & 0.0377 & 0.0726 & 0.0529 \\
\hline \multirow{2}{*}{2.6} & $0.8895 \pm$ & $0.4087 \pm$ & $0.6989 \pm$ & $1.1932 \pm$ & $1.3224 \pm$ \\
\hline & 0.0687 & 0.0480 & 0.0550 & 0.0577 & 0.0576 \\
\hline \multirow{2}{*}{2.8} & $0.9317 \pm$ & $0.3767 \pm$ & $0.7423 \pm$ & $1.5161 \pm$ & $1.1242 \pm$ \\
\hline & 0.0923 & 0.0716 & 0.1107 & 0.0622 & 0.0992 \\
\hline \multirow{2}{*}{3.0} & $0.8367 \pm$ & $0.3090 \pm$ & $0.5415 \pm$ & $1.5875 \pm$ & $1.1293 \pm$ \\
\hline & 0.0833 & 0.0517 & 0.0702 & 0.0502 & 0.0642 \\
\hline \multirow{2}{*}{3.2} & $1.0070 \pm$ & $0.5878 \pm$ & $0.5338 \pm$ & $1.6129 \pm$ & $1.1183 \pm$ \\
\hline & 0.0799 & 0.0769 & 0.0771 & 0.0570 & 0.0785 \\
\hline \multirow{2}{*}{3.4} & $0.8430 \pm$ & $0.5574 \pm$ & $0.5512 \pm$ & $1.5478 \pm$ & $1.1180 \pm$ \\
\hline & 0.0686 & 0.0721 & 0.0815 & 0.0534 & 0.0694 \\
\hline
\end{tabular}

Table 11. Examples of $t_{p}$ mean values and $95 \%$ confidence intervals $(\mathrm{Cl})$ determined for one heel-strike pattern runner in frequency range $f_{r}=2.4-3.4 \mathrm{~Hz}$ (results of laboratory tests)

\begin{tabular}{|c|c|c|c|c|c|}
\hline \multirow{2}{*}{$\begin{array}{c}\text { Frequency } f_{r \prime} \\
\mathrm{Hz}\end{array}$} & \multicolumn{5}{|c|}{ Amplitudes of the Gaussian functions $A_{p}$} \\
\hline & $t_{1} \pm \mathrm{Cl}$ & $t_{2} \pm \mathrm{Cl}$ & $t_{3} \pm \mathrm{Cl}$ & $t_{4} \pm \mathrm{Cl}$ & $t^{5} \pm \mathrm{Cl}$ \\
\hline \multirow{2}{*}{2.4} & $0.0337 \pm$ & $0.0504 \pm$ & $0.0813 \pm$ & $0.1359 \pm$ & $0.2086 \pm$ \\
\hline & 0.00098 & 0.00234 & 0.00273 & 0.00264 & 0.00413 \\
\hline \multirow{2}{*}{2.6} & $0.0305 \pm$ & $0.0471 \pm$ & $0.0793 \pm$ & $0.1307 \pm$ & $0.1982 \pm$ \\
\hline & 0.00063 & 0.00218 & 0.00262 & 0.00249 & 0.00404 \\
\hline \multirow{2}{*}{2.8} & $0.0319 \pm$ & $0.0513 \pm$ & $0.0820 \pm$ & $0.1330 \pm$ & $0.1989 \pm$ \\
\hline & 0.00096 & 0.00232 & 0.00295 & 0.00404 & 0.00604 \\
\hline \multirow{2}{*}{3.0} & $0.0298 \pm$ & $0.0444 \pm$ & $0.0703 \pm$ & $0.1171 \pm$ & $0.1844 \pm$ \\
\hline & 0.00101 & 0.00344 & 0.00432 & 0.00322 & 0.00514 \\
\hline \multirow{2}{*}{3.2} & $0.0305 \pm$ & $0.0496 \pm$ & $0.0766 \pm$ & $0.1105 \pm$ & $0.1728 \pm$ \\
\hline & 0.00074 & 0.00192 & 0.00205 & 0.00332 & 0.00483 \\
\hline \multirow{2}{*}{3.4} & $0.0317 \pm$ & $0.0487 \pm$ & $0.0745 \pm$ & $0.1056 \pm$ & $0.1640 \pm$ \\
\hline & 0.00127 & 0.00255 & 0.00319 & 0.00313 & 0.00392 \\
\hline
\end{tabular}


Table 12. Examples of $\sigma_{p}$ mean values and $95 \%$ confidence intervals $(\mathrm{Cl})$ determined for one heel-strike pattern runner in frequency range $f_{r}=2.4-3.4 \mathrm{~Hz}$ (results of laboratory tests)

\begin{tabular}{|c|c|c|c|c|c|}
\hline \multirow{2}{*}{$\begin{array}{c}\text { Frequency } f_{r \prime} \\
\mathrm{Hz}\end{array}$} & \multicolumn{5}{|c|}{ Amplitudes of the Gaussian functions $A_{p}$} \\
\hline & $\sigma_{1} \pm \mathrm{Cl}$ & $\sigma_{2} \pm \mathrm{Cl}$ & $\sigma_{3} \pm \mathrm{Cl}$ & $\sigma_{4} \pm \mathrm{Cl}$ & $\sigma_{5} \pm \mathrm{Cl}$ \\
\hline \multirow{2}{*}{2.4} & $0.0100 \pm$ & $0.0177 \pm$ & $0.0315 \pm$ & $0.0491 \pm$ & $0.0777 \pm$ \\
\hline & 0.00043 & 0.00117 & 0.00096 & 0.00154 & 0.00351 \\
\hline \multirow{2}{*}{2.6} & $0.0089 \pm$ & $0.0176 \pm$ & $0.0314 \pm$ & $0.0461 \pm$ & $0.0639 \pm$ \\
\hline & 0.00032 & 0.00113 & 0.00083 & 0.00136 & 0.00204 \\
\hline \multirow{2}{*}{2.8} & $0.0095 \pm$ & $0.0169 \pm$ & $0.0316 \pm$ & $0.0434 \pm$ & $0.0515 \pm$ \\
\hline & 0.00031 & 0.00089 & 0.00232 & 0.00137 & 0.00165 \\
\hline \multirow{2}{*}{3.0} & $0.0090 \pm$ & $0.0156 \pm$ & $0.0266 \pm$ & $0.0408 \pm$ & $0.0505 \pm$ \\
\hline & 0.00052 & 0.00152 & 0.00202 & 0.00126 & 0.00141 \\
\hline \multirow{2}{*}{3.2} & $0.0086 \pm$ & $0.0160 \pm$ & $0.0207 \pm$ & $0.0394 \pm$ & $0.0453 \pm$ \\
\hline & 0.00026 & 0.00068 & 0.00160 & 0.00129 & 0.00198 \\
\hline \multirow{2}{*}{3.4} & $0.0087 \pm$ & $0.0162 \pm$ & $0.0207 \pm$ & $0.0375 \pm$ & $0.0432 \pm$ \\
\hline & 0.00032 & 0.00116 & 0.00168 & 0.00110 & 0.00127 \\
\hline
\end{tabular}

Analysing the CI values presented in Tables 10-12, relatively significant changes in $A_{p}$ values and only minor changes in $t_{p}$ and $\sigma_{p}$ values can be observed. Similar results were achieved by Racic \& Morin (2014), who proposed the method of using VGRF/G templates and presented the parameters of the elaborated template with $95 \%$ confidence intervals.

In case of modelling the $V G R F / G$ using the Gaussian functions, it is essential to take into account the primary general rule observed during $V G R F$ measurements, this is, that the increase in the amplitude of the VGRF is associated with a decrease in the value of the contact time $t_{c}$ (in other words, when the peak amplitude of the template increases, the value of the contact time $t_{c}$ decreases and vice versa). This link is visible in Figure 14 for a wide range of running frequency, but the same general rule applies to the changes in the values of the $V G R F / G$ generated by one person for each running frequency. Moreover, other parameters of the Gaussian functions should be changed according to the trends presented in Figure 13.

\section{Summary and conclusions}

The issue of footbridge vibration is usually considered in case of footbridges loaded by pedestrian groups or a crowd. However, sizable vibrations of footbridges can be induced by just one running person. 
These excessive vibrations can be a serious problem depending on the location of the footbridge. The dynamic action of runners can be particularly significant in case of footbridges located in the vicinity of large public transport stations (train or metro stations, bus or tram stops), as well as in case of footbridges located in recreational areas (city parks, walking boulevards or promenades). In these places, the risk of the presence of people running (hurrying or exercising (jogging)) is high. The appropriate technique for the modelling of the dynamic loads generated by people running is crucial to determine the correct value of the dynamic response of the structure.

Different methods of modelling of the amplitude of the normalised vertical ground reaction force presented in the paper allow accurate prediction of the vertical ground reaction force values which, consequently, enable an increase in the accuracy of dynamic analyses of the structures subjected to the dynamic action of people running. Considering the results of laboratory measurements of the vertical ground reaction force, the improvements of the models of ground reaction forces proposed by different authors were presented. Two running techniques - forefoot strike running and heel-strike running were taken into consideration and the new model of the vertical ground reaction force for the heel-strike running technique was proposed. Furthermore, an important improvement of the model was elaborated in the form of equations defining the contact time of the foot with the ground during running as a function of frequency of running for the forefoot strike and heel-strike running technique separately. All proposed improvements address the shortages and shortcomings in this area and, consequently, contribute to increasing the accuracy of determining the vertical ground reaction force values.

In addition, an extensive description of the amplitude of the normalised vertical ground reaction force modelling technique through the Gaussian functions has been presented. The results of analyses of the variability of the Gaussian function parameters as a function of running frequency have been shown. The equations describing the Gaussian function parameters are given. The proposed equations allow easy and accurate determination of the amplitude of the normalised vertical ground reaction force template for any running frequency, which facilitates the practical implementation of the method. Notable findings include the trends of variability of the Gaussian function parameters with increased frequency of running and the general conclusion describing the dependency of the vertical ground reaction force amplitude and contact time of the foot with the ground, in other words, when the amplitude of the vertical ground reaction force increases, the contact time of the foot with the ground decreases. This 
general conclusion should be considered during the modelling of the vertical ground reaction force generated by one person running with a given frequency to reproduce the variability (random changes) of the vertical ground reaction force.

The described modelling techniques used together with the information on the variability of the amplitude of the normalised vertical ground reaction force parameters (the variability of the amplitude of the normalised vertical ground reaction force curves indicators) allow generation of the random amplitude of the normalised vertical ground reaction force values. Consideration of the random changes of the vertical ground reaction force parameters will allow further improvement of the accuracy of the dynamic analyses.

\section{Acknowledgements}

The author would like to acknowledge all volunteers (students from the University of Physical Education in Cracow (UPE) and Cracow University of Technology (CUT)), as well as employees of the Department of Biomechanics of the UPE and employees of the Division of Bridge, Metal and Timber Structures of the CUT for participation in the laboratory tests and their input in data collection and all those who helped to prepare the collected data for further analysis.

\section{Funding}

This work was supported by the Faculty of Civil Engineering of the Cracow University of Technology under Dean's Grant.

\section{REFERENCES}

Almeida, M. O., Saragiotto, B. T., Yamato, T. P., \& Lopes, A. D. (2015). Is the rearfoot pattern the most frequently foot strike pattern among recreational shod distance runners? Physical Therapy in Sport, 16(1), 29-33. http://dx.doi.org/10.1016/j.ptsp.2014.02.005

Bachmann, H., Ammann, W., Deischl, F., et al. (1995). Vibration Problems in Structures: Practical Guideline. Basel: Birkhäuser.

Bakkie, D., Mache, M. A., Johnson, C., Garcia-Krauss, P., Kendall, K., \& Hsieh, C. (2013). Foot strike pattern trends in sub-elite half marathon runners. In 31 International Conference on Biomechanics in Sports. https://ojs.ub.uni-konstanz.de/cpa/article/view/5578

Clark, K. P., \& Weyand, P. G. (2014). Are running speeds maximized with simple-spring stance mechanics?. Journal of Applied Physiology, 117(6), 604-615. https://doi.org/10.1152/japplphysiol.00174.2014 
Gruber, A. H., Davis, I. S., \& Hamill, J. (2011). Frequency content of the vertical ground reaction force component during rearfoot and forefoot running patterns. Medicine \& Science in Sports \& Exercise, 43(5).

https://doi.org/10.1249/01.MSS.0000402852.25234.f0

Gruber, A. H., Edwards, W. B., Hamill, J., Derrick, T. R., \& Boyer, K. A. (2017). A comparison of the ground reaction force frequency content during rearfoot and non-rearfoot running patterns. Gait \& Posture, 56, 54-59.

https://doi.org/10.1016/j.gaitpost.2017.04.037

Hamill, J., \& Gruber, A. H. (2017). Is changing footstrike pattern beneficial to runners? Journal of Sport and Health Science, 6(2), 146-153. https://doi.org/10.1016/j.jshs.2017.02.004

Hasegawa, H., Yamauchi, T., \& Kraemer, W. J. (2007). Foot strike patterns of runners at the 15-km point during an elite-level half marathon. Journal of Strength and Conditioning Research, 21(3), 888-893.

https://doi.org/10.1519/00124278-200708000-00040

Larson, P., Higgins, E., Kaminski, J., Decker, T. Preble, J., Lyons, D., McIntyre K., \& Normile, A. (2011). Foot strike patterns of recreational and sub-elite runners in a long-distance road race. Journal of Sports Sciences, 29(15), 1665-1673. http://dx.doi.org/10.1080/02640414.2011.610347

Nilsson, J., \& Thorstensson, A. (1989). Ground reaction forces at different speeds of human walking and running. Acta Physiologica Scandinavica, 139, 217-227. https://doi.org/10.1111/j.1748-1716.1989.tb08655.x

Occhiuzzi, A., Spizzuoco, M., \& Ricciardelli, F. (2008). Loading models and response control of footbridges excited by pedestrians. Structural Control and Health Monitoring, 15(3), 349-368. https://doi.org/10.1002/stc.248

Racic, V., \& Morin, J. B. (2014). Data-driven modelling of vertical dynamic excitation of bridges induced by people running. Mechanical Systems and Signal Processing, 43(1-2), 153-170. https://doi.org/10.1016/j.ymssp.2013.10.006

Rainer, J. H., \& Pernica, G. (1986). Vertical dynamic forces from footsteps. Canadian Acoustics, 14(2), 12-21. https://jcaa.caa-aca.ca/index.php/jcaa/article/view/559

Rainer, J. H., Pernica, G., \& Allen, D. E. (1988). Dynamic loading and response of footbridges. Canadian Journal of Civil Engineering, 15(1), 66-71. https://doi.org/10.1139/188-007

Scheerder, J., Breedveld, K., \& Borgers, J. (2015). Running across Europe. The rise and size of one of the largest sport markets. London: Palgrave Macmillan. https://doi.org/10.1057/9781137446374

Sołtys, M., Birnbaum, A., Jeleniewski, P., et al. (2014). Report Poland Runs National list of runners 2014 (Raport Polska Biega - Narodowy Spis Biegaczy 2014), Agora SA, Warsaw (in Polish).

Wheeler, J. E., (1982). Prediction and control of pedestrian induced vibration in footbridges. ASCE Journal of the Structural Division, 109(9), 2045-2065. 


\section{NOTATIONS}

$\alpha_{i}-$ dynamic load factors (Fourier coefficients)

$\alpha_{\varphi}$-impact peak location coefficient

$\beta_{\varphi}$ - impact peak slenderness

$\lambda$-impact peak time-range coefficient $(\lambda=0.17)$

$\zeta$ - damping ratio

$\sigma_{p}-$ width of the Gaussian function (also called Gaussian RMS width)

$\varphi_{i}$ - phase shifts of the $i^{\text {th }}$ harmonics with respect to the $1^{\text {st }}$ harmonic

$\varphi_{i p}(t)$ - function of impact peak (Eq. (6))

$\Phi(t)$ - vertical ground reaction force determined by means of Eq. (7)

$\Omega(t)$ - normalised vertical ground reaction force $(V G R F / G)$ interpolated by means of the Gaussian functions (Eq. (9))

$A_{\varphi}$ - amplitude of the impact peak (Eq. (6))

$A_{i p}$ - normalised (dimensionless) amplitude of the impact peak during heel-strike running

$A_{p}$ - amplitude (height) of the Gaussian function (Eq. (9))

$A_{p p, h}$ or $A_{p p, f}$ - the normalised (dimensionless) amplitude of the propulsive peak in case of heel-strike running $\left(A_{p p, h}\right)$ and forefoot strike running $\left(A_{p p, f}\right)$

$A_{r}$ - dynamic amplification factor (Eq. (4))

$F(t)$ - vertical ground reaction force determined by means of Eq. (1) or (2)

$F_{a v}$ - mean value of normalised force $F_{a v}=J_{G} / t_{c}$ (see Table 3)

$f_{r}$ - step frequency during running (running frequency)

$G$ - body weight of the running person

$i$ - order number of the harmonic $i=1,2,3, \ldots$

$j$ - time range coefficient, $j=0,1,2,3, \ldots$ (Eq. (2))

$J_{G}$ - normalised impulse of force (area under the normalised $V G R F / G$ graph, see Table 3)

$k$ - contact time factor (see Eq. (3))

$n$ - total number of included harmonics (for running, the first three harmonics are usually considered, $n=3$ )

$p$ - total number of the included Gaussian functions, (Eq. (9), $p=5$ )

$t$ - time step

$t_{c}$ - contact time of the foot with the ground, $t_{c}=t_{c, f}$ or $t_{c}=t_{c, h}$ for forefoot or heel-strike running, respectively

$t_{c h, a}$ or $t_{c f, a}$ - approximated contact time of the foot with the ground for heel-strike running and forefoot strike running, respectively

$t_{i p}$ - time of occurrence of $A_{i p}$

$t_{p}$ - position of the peak of the Gaussian function

$t_{p p, h}$ and $t_{p p, f}$-time of occurrence of $A_{p p, h}$ and $A_{p p, f}$, respectively

$T_{r}$ - running period, $T_{r}=1 / f_{r}$

SD - standard deviation

CI - 95\% confidence interval 